\title{
Equitable decision making approaches over allocations of multiple benefits to multiple entities
}

\author{
Nur Kaynar, Özlem Karsu* \\ Industrial Engineering Department, Bilkent University, Ankara, Turkey
}

\section{A R T I C L E I N F O}

\section{Article history:}

Received 10 February 2017

Accepted 5 October 2017

Available online 7 October 2017

\section{Keywords:}

Interactive approaches

Additive utility

Convex cone method

Fairness

Equity

\begin{abstract}
A B S T R A C T
In this study, we develop decision support tools for policy makers that will help them make choices among a set of allocation alternatives. We assume that alternatives are evaluated based on their benefits to different users and that there are multiple benefit (output) types to consider. We assume that the policy maker has both efficiency (maximizing total output) and equity (distributing outputs across different users as fair as possible) concerns. This problem is a multicriteria decision making problem where the alternatives are represented with matrices rather than vectors.

We develop interactive algorithms that guide a policy maker to her most preferred solution, which are based on utility additive (UTA) and convex cone methods. Our computational experiments demonstrate the satisfactory performance of the algorithms. We believe that such decision support tools may be of great use in practice and help in moving towards fair and efficient allocation decisions.
\end{abstract}

(c) 2017 Elsevier Ltd. All rights reserved.

\section{Introduction}

In many decision making settings the decision makers have to choose among a set of given alternatives, considering multiple criteria. Due to the trade-offs that exist between different criteria, this choice problem is a challenging problem, which motivated the design of many decision support tools (see [1] for a survey). Some example settings from the literature involve decision making problems in the areas of energy planning [2,3] finance [4], and sustainability $[5,6]$. In such settings, each alternative is associated with a vector whose elements show the performance of that alternative with respect to each criterion. These problems are multiple criteria evaluation problems where a finite set of alternatives is given explicitly. For evaluation problems, one may try to: identify the best alternative or a small subset of most preferred alternatives, rank the alternatives, or sort the alternatives into predefined groups [7].

A special multicriteria choice problem occurs when the alternatives are allocation vectors, in which each element corresponds to the amount of a benefit that a beneficiary enjoys [8]. In such cases, the DM has to choose the best allocation considering both fairness (the concern for choosing an allocation as fair as possible) and efficiency (the concern for choosing an allocation that has high total benefit). The tradeoff between these two concerns makes the problem challenging. For example, considering two alternative

\footnotetext{
th This manuscript was processed by Associate Editor Dias.

* Corresponding author.

E-mail address: ozlemkarsu@bilkent.edu.tr (Ö. Karsu).
}

allocations of a good across two beneficiaries: $(5,6)$ and $(3,10)$, one can say that the first one is fairer, while the second one is more efficient. Some examples of such equitable choice problems are public service facility location problems, in which the best location is to be chosen among candidate locations (efficiency is the desire to choose the alternative that minimizes the total distance to the users, fairness is the desire to be equally close to all users) and healthcare project selection problems, in which each project is associated with the distribution of a health gain across different population groups (efficiency is the concern of maximizing the total health gain and fairness is the desire to be fair in the health gain distribution). A distinguishing feature of such choice problems is the impartiality property, which assumes that the identities of the beneficiaries are not important and do not affect the decision. In such a setting the decision maker is indifferent between an allocation and any permutation of that allocation, making the problem and the solution approaches different than their counterparts in the classical multicriteria decision making literature (see [8-11] and references therein).

In many real life problems, there are multiple beneficiaries enjoying multiple benefits, hence efficiency and fairness concerns occur on multiple dimensions. In this study, we address such settings and consider multicriteria decision making (MCDM) problems, where the alternatives are not vectors but matrices, the columns and rows of which show the allocated outputs and the users that enjoy these goods, respectively. Each element of the matrix shows the level of an output a user receives. In this sense this 
problem generalizes the two problems (the classical MCDM choice problem) and (the equitable choice problem) mentioned above.

In this work we consider a choice problem in which a policy maker (decision maker) is faced with a set of distribution alternatives that are evaluated with respect to how multiple outputs are distributed across multiple users. Such allocation problems are encountered in many real life cases; however our initial motivation was policy making decisions in healthcare, which show the tradeoff between fairness and efficiency to a very high degree. In such settings alternative projects/project portfolios are evaluated not only based on total gain (e.g. total increase in the qualityadjusted life years of the population) but also based on how this gain will be distributed to different population groups. Moreover, health gain is usually not the only outcome of interest, other outcomes such as protection from healthcare related financial catastrophes or decrease in out-of-pocket expenses are also considered $[12,13]$.

This problem can be considered as related to the group decision making problem, where alternatives that have different consequences for a number of entities (individuals) are evaluated, typically by the group of entities itself. Hence in such settings, one of the main concerns is constructing a social welfare function whose arguments are the individual utilities. The suggested decision support methods include assessments of the preferences of individuals and a rule for aggregating these preferences to determine group preferences $[14,15]$. The pioneering studies that deal with aggregation of cardinal utilities are due to [14,16-19]. Recently, Greco et al. [20] proposed an extension of the robust ordinal regression method to multiple criteria group decision problems.

One of the important concerns in group decision making is equity (fairness) of the group decision [21-24]. In line with this, Eliashberg and Winkler [22] structure a framework in which an individual's utility depends on what others receive. Group members' approach to equity is reflected through individual utility functions, which are functions of the distribution vector. Similar to the previous studies, the authors consider a linear aggregation rule. Keeney [23] considers equity in distributions of risk and Harvey [24] extends this discussion by considering preferences on trade-offs and develops notions of inequity neutrality and inequity aversion. He discusses different conditions and links them to various forms of group value functions.

In most of the group decision making studies, individuals have different preference models (represented by different individual utility functions) and the aim is aggregating these preferences into a group preference model. However, in the problem settings we consider, we assume that there is a single policy maker (DM) hence we do not have the concern of aggregating individual preferences. In group decision making, since each individual's utility is usually considered as a function of what he receives (independent of what others get), assuming an additive social welfare function may be realistic. As we will elaborate later, we try to relax the preferential separability assumption, which is common to many group decision making settings, since we assume that the policy maker's preferences involve equity concerns (hence will depend on how a benefit is distributed) alongside efficiency concerns. Even when separability is assumed, we structure the framework so as to encourage equity in the distributions of benefits.

\section{Problem definition}

Consider an example healthcare project selection problem in which the policy maker is to choose a project to initiate among a set of projects. In this problem, we are given a set of alternatives $A=\left\{a^{1}, a^{2}, \ldots, a^{N}\right\}$ and a typical member shows the distribution of multiple $(n)$ outputs over multiple $(m)$ users. In the matrix representation, the rows and columns correspond to different users (population groups) and outputs, respectively as follows:

$$
a^{k}=m \text { Users }\left[\begin{array}{cccc}
a_{11}^{k} & a_{12}^{k} & \cdots & a_{1 n}^{k} \\
a_{21}^{k} & a_{22}^{k} & \cdots & a_{2 n}^{k} \\
\vdots & \vdots & \ddots & \vdots \\
a_{m 1}^{k} & a_{m 2}^{k} & \cdots & a_{m n}^{k}
\end{array}\right]
$$

where for a given alternative $a^{k}, a_{i j}^{k}$ represents the level of output $j$ allocated to user $i$. We assume that the decision maker is trying to select the best alternative in line with her preferences.

This problem can be considered as a multicriteria choice problem, in which alternatives are explicitly given and the problem is determining the most preferred one. However, it is an MCDM problem of a special type. It is different than the classical MCDM problems discussed in the literature in the sense that the alternatives correspond to matrices rather than vectors.

Moreover, unlike a classical MCDM problem, this problem involves fairness factors alongside the usual trade-off between different outputs. That is, how we distribute outputs is also of concern to the decision maker. We will try to explain the relation and the possible trade-off between equity and efficiency by using a small example.

Example 1. Consider a problem in which a DM is faced with a set of alternatives showing distributions of two outputs to two users. When we increase efficiency with respect to both outputs, that is when we increase the total amount distributed in both output 1 and output 2, while keeping the equity levels same, we obtain a better alternative. For example, when we have $\left(\begin{array}{ll}5 & 5 \\ 5 & 5\end{array}\right)$ and $\left(\begin{array}{ll}6 & 6 \\ 6 & 6\end{array}\right)$ as two alternatives, the DM will choose $\left(\begin{array}{ll}6 & 6 \\ 6 & 6\end{array}\right)$ over $\left(\begin{array}{ll}5 & 5 \\ 5 & 5\end{array}\right)$, since $\left(\begin{array}{ll}6 & 6 \\ 6 & 6\end{array}\right)$ distributes higher amounts of outputs to the users and both alternatives have complete equality. This example illustrates the efficiency concerns of the DM.

When we have a more equitable allocation in both goods while keeping the efficiency levels same, we obtain a better alternative. For example, when we have $\left(\begin{array}{ll}3 & 3 \\ 5 & 5\end{array}\right)$ and $\left(\begin{array}{ll}4 & 4 \\ 4 & 4\end{array}\right)$ as two alternatives, the DM will choose $\left(\begin{array}{ll}4 & 4 \\ 4 & 4\end{array}\right)$ over $\left(\begin{array}{ll}3 & 3 \\ 5 & 5\end{array}\right)$. Both alternatives have the same efficiency levels with respect to both outputs, they distribute 8 units of output 1 and 8 units of output 2, but alternative 2 provides a more equitable allocation for each of the outputs.

In the first example, only efficiency levels change and in the second example, only equity levels change. Therefore, they do not reflect the trade-off between the two concerns that many real life examples come along with. Choosing between alternatives where both efficiency and equity levels change can be a cognitively challenging task. For example, we cannot say which alternative would be chosen between $\left(\begin{array}{ll}4 & 5 \\ 6 & 7\end{array}\right)$ and $\left(\begin{array}{ll}3 & 4 \\ 8 & 9\end{array}\right)$. In this example, the alternative that has higher efficiency levels is worse in terms of equity. The trade-off between equity and efficiency can be observed here. Note that even this example is insufficient to reflect the challenge to its full extent, since the second alternative is more efficient and less equitable with respect to both outputs. In fact, the problem involves multidimensional efficiency and multidimensional equity concerns since multiple outputs are distributed. Consider $\left(\begin{array}{ll}4 & 4 \\ 6 & 9\end{array}\right)$ and $\left(\begin{array}{ll}3 & 5 \\ 8 & 7\end{array}\right)$ : the second alternative is more efficient (and less equitable) with respect to output 1 while the first alternative is more efficient (and less equitable) with respect to output 2.

In this study we assume a non-hierarchical relation among the users. We assume that changing the bundles over the users does 
not affect the social welfare value that alternative brings. (A bundle is a distribution of benefits to a single user and corresponds to a row in our matrix notation). This is the so-called impartiality assumption defined in equitable preferences [25]. For example, we assume that the DM will be indifferent between two alternatives $\left(\begin{array}{ll}4 & 5 \\ 3 & 6\end{array}\right)$ and $\left(\begin{array}{ll}3 & 6 \\ 4 & 5\end{array}\right)$

As seen in Example 1 the efficiency and fairness concerns are of a multidimensional nature as we consider situations in which multiple benefits are distributed to multiple entities. There are wellknown results in the economics literature on single benefit (income) distributions to multiple individuals that discuss various axioms and link these to dominance relations such as Lorenz dominance [26] and Generalized Lorenz dominance [27]. However, it is considerably harder to obtain such rules and equivalence results in a multidimensional framework [28]. A pioneering work that touches upon these dominance issues in the multidimensional settings is due to [29]. We also provide dominance rules in line with the assumptions (impartiality and monotonicity) that we make on the preference model of the central DM.

These dominance rules are obtained by extending vector dominance relations for alternatives that are represented by matrices. We will first give the definition of (weak) dominance relation over vectors and then, discuss the corresponding extensions.

Definition 1. Given two alternatives $z^{k}, z^{k^{\prime}} \in \mathbb{R}^{n}$ where $\mathrm{n}$ is the number of outputs (criteria) and $J=\{1,2, \ldots, n\}$,

$$
z^{k} \preceq_{d} z^{k^{\prime}}\left(z^{k^{\prime}} \text { weakly dominates } z^{k}\right) \Longleftrightarrow z_{j}^{k} \leq z_{j}^{k^{\prime}} \text { for all } j \in J \text {. }
$$

A simple extension of Definition 1 for our problem setting would be the following:

Definition 2. Given two alternatives $a^{k}, a^{k^{\prime}} \in \mathbb{R}^{(m x n)}$ where $\mathrm{m}$ and $\mathrm{n}$ are the number of users and the number of outputs, respectively, let us define the following sets $I=\{1,2, \ldots, m\}$ and $J=\{1,2, \ldots, n\}$

$a^{k} \preceq_{d} a^{k^{\prime}}\left(a^{k^{\prime}}\right.$ weakly dominates $\left.a^{k}\right) \Longleftrightarrow a_{i j}^{k} \leq a_{i j}^{k^{\prime}}$ for all $\mathrm{i} \in I, \mathrm{j}$ $\in J$

Consider two alternatives $a^{k}=\left(\begin{array}{ll}5 & 4 \\ 4 & 3\end{array}\right)$ and $a^{k^{\prime}}=\left(\begin{array}{ll}6 & 5 \\ 4 & 3\end{array}\right)$. Since $a_{i j}^{k} \leq a_{i j}^{k^{\prime}}$ for all $i, j$, we say that $a^{k^{\prime}}$ dominates $a^{k}$. Alternative $k^{\prime}$ brings greater value to the first user for each criterion than alternative $k$ while the second user gets the same bundle in both alternatives. Here, the users are called as first and second just to provide an ease in the expression. Their usage do not imply any superiority relation. Let us consider a scenario where alternative $k$ becomes $a^{k}=\left(\begin{array}{ll}4 & 3 \\ 5 & 4\end{array}\right)$. From the impartiality assumption, the DM is indifferent between $\left(\begin{array}{ll}5 & 4 \\ 4 & 3\end{array}\right)$ and $\left(\begin{array}{ll}4 & 3 \\ 5 & 4\end{array}\right)$. However, the dominance rule introduced in Definition 2 fails to acknowledge this relation when the row ordering of the users changes. Hence, we modify this dominance rule to handle the impartiality assumption.

Definition 3. For an alternative $a^{k} \in \mathbb{R}^{(m x n)}$ where $m$ and $n$ represent the number of users and the number of outputs, respectively, let $\pi\left(a^{k}\right)$ be the set of all different row permutations of $a^{k}$ and $R=\{1,2, \ldots, m !\}$. Given two alternatives $a^{k}$ and $a^{k^{\prime}} \in \mathbb{R}^{(m \times n)}$,

$a^{k} \preceq_{e m} a^{k^{\prime}} \quad\left(a^{k^{\prime}}\right.$ equitably matrix weak dominates (emdominates) $\left.a^{k}\right) \Longleftrightarrow \pi_{r}\left(a^{k}\right) \preceq_{d} a^{k^{\prime}}$ for at least one $r \in R$.

Em-dominance enables us to make further inferences compared to the previous dominance relations. Let us take the example where $a^{k}=\left(\begin{array}{ll}4 & 3 \\ 5 & 4\end{array}\right)$ and $a^{k^{\prime}}=\left(\begin{array}{ll}6 & 5 \\ 4 & 3\end{array}\right)$ and $\pi\left(a^{1}\right)=$ $\left\{\left(\begin{array}{ll}4 & 3 \\ 5 & 4\end{array}\right),\left(\begin{array}{ll}5 & 4 \\ 4 & 3\end{array}\right)\right\}$. Since $\pi_{2}\left(a^{k}\right)=\left\{\left(\begin{array}{ll}5 & 4 \\ 4 & 3\end{array}\right)\right\}$ and $\pi_{2}\left(a^{k}\right) \preceq_{d}$ $a^{k^{\prime}}, a^{k^{\prime}}$ em-dominates $a^{k}$. The em-dominance relation will help us eliminate some alternatives. However, in most real life cases, we will have trade-offs and using dominance relations will not be sufficient to make decisions. Hence we propose decision support tools, that will help the DM choose her most preferred alternative in a set of em-efficient alternatives (an alternative is em-efficient if there is no other alternative that em-dominates it).

\section{Solution approaches}

Recall that we consider the problem of selecting the best alternative among a finite set of alternatives. In the literature, different approaches such as outranking relations and multi-attribute value theory approaches are used for this problem type [30]. We propose value function based solution approaches to this problem. Such approaches assume that the DM's preferences can be represented by value functions. We construct our approaches by defining three different value functions: marginal value function (MVF), bundle value function (BVF) and social welfare function (SWF).

For each output a MVF is defined, which assigns value scores to different levels of the output. Let $M V_{j}($.$) be the non-decreasing$ marginal value function for output $j . M V_{j}\left(a_{i j}^{k}\right)$ represents the value derived by the DM (policy maker) from the allocation of the $j$ th output of alternative $k$ to any user $i$. Hence, MVF depends only on the output type and not the user enjoying it.

Another function that can be defined is the bundle value function (BVF). Let $B V\left(b_{i}^{k}\right)$ be the social value (as perceived by the DM) derived by providing a user with bundle $b_{i}^{k}$ (this is the $i$ th row in alternative $k$ ) (see $[28,29]$ for further discussion on this assumption). In other words, $B V($.) assigns a total value score to the bundles (vectors showing levels of output with respect to all output types). Again, due to impartiality, we assume that this value does not depend on users' identities.

We also define a social welfare function (SWF) for the alternatives. Let $S W\left(a^{k}\right)$ be the total social welfare that alternative $k$ brings. It will be used to evaluate overall values of the alternatives to the DM.

Example 2. To illustrate these functions, let us consider an example problem where a healthcare policy maker aims to choose the best alternative among the six alternatives provided below. Let the alternatives correspond to different distributions of two outcomes, increase in quality adjusted life time and decrease in out-of-pocket expenditures, to two user groups. Hence two MVFs will be defined: one for the increase in quality adjusted life time and one for the decrease in out-of-pocket expenditures $\left(M V_{1}(\right.$.$) and M V_{2}()$. for short.)

$$
\begin{aligned}
& a^{1}=\left(\begin{array}{ll}
2 & 8 \\
3 & 4
\end{array}\right) a^{2}=\left(\begin{array}{ll}
5 & 5 \\
6 & 2
\end{array}\right) a^{3}=\left(\begin{array}{ll}
4 & 6 \\
3 & 5
\end{array}\right) a^{4}=\left(\begin{array}{ll}
5 & 5 \\
4 & 6
\end{array}\right) \\
& a^{5}=\left(\begin{array}{ll}
3 & 5 \\
8 & 2
\end{array}\right) a^{6}=\left(\begin{array}{ll}
6 & 4 \\
3 & 7
\end{array}\right)
\end{aligned}
$$

In this example, the levels for outputs 1 and 2 are $(2,3,4,5,6,8)$ and $(2,4,5,6,7,8)$, respectively. MVFs convert these levels into their value correspondences. For example, $M V_{1}(6)$ and $M V_{2}(5)$ represent the values obtained from getting 6 and 5 units from the first and the second outputs, respectively. Each user receives a bundle of two outputs (e.g. user 1 gets $(2,8)$ in the first alternative and $(5,5)$ in the second alternative) and BVFs calculate the total value (as perceived by DM) that a bundle brings to a user (e.g. $\operatorname{BV}(2,8)$ returns the value that bundle $(2,8)$ brings). Similarly, SWFs assign total values to the alternative (e.g. $\operatorname{SW}\left(\left(\begin{array}{ll}2 & 8 \\ 3 & 4\end{array}\right)\right)$ gives the social welfare value that the first alternative brings).

The methods we discuss below are based on different assumptions on the forms of these marginal value, bundle value, and social welfare functions, which are summarized in Table 1 . The first 
Table 1

Summary of the solution approaches.

\begin{tabular}{lllll}
\hline Approach & MVF & BVF & SWF & Preference information \\
\hline UTA-based & Concave & Additive & Additive & Vector comparisons \\
Cone-based & Linear & Additive & S. quasi-concave & Holistic comparisons \\
\hline
\end{tabular}

approach exploits UTA techniques and uses concave MVFs to encourage equitable distribution of an output. BVFs are assumed to be additive, i.e. the total value that a user acquires through an alternative is the sum of the values that she obtains from each output. Furthermore, social welfare that an alternative brings is assumed to be the sum of bundle values. The second approach (convex cone based approach) assumes linear MVFs and additive BVFs, which are weighted aggregations of the marginal values of the outputs. This approach also assumes SWFs are symmetric quasiconcave and hence it relaxes the additivity assumption of the first approach.

We design interactive algorithms that take preference information from the DM iteratively by asking pairwise comparison questions. In the UTA-based approach, we ask the DM to compare two different bundles of outputs (vectors) while the convex cone based approach asks the DM to compare alternatives holistically. As a small example, when holistic comparison method is employed, the DM is asked to compare two alternatives from the given set such as $\left(\begin{array}{ll}2 & 8 \\ 3 & 4\end{array}\right)$ and $\left(\begin{array}{lr}5 & 5 \\ 6 & 2\end{array}\right)$ whereas when vector (bundle) comparison method is employed, the DM is asked to compare the following bundles $(3,4)$ and $(6,2)$, which represent different distributions of outputs to only one user. Holistic comparison is more challenging in terms of its cognitive requirements. However one can eliminate the alternative which is not preferred, permanently from the set. Vector comparison questions are easier for the DM but we cannot eliminate any alternative directly based on such questions.

\subsection{UTA-based method}

In this part, we discuss the interactive approach based on the well-known UTA method introduced by Jacquet-Lagrèze and Siskos [31,32]. In a classical MCDM problem, this method assumes an additive unweighted global value function, which is the sum of the marginal value scores and assigns values to alternatives in line with the preferences of a DM by using linear programming techniques [33-35]. Although the marginal value functions are forced to be compatible with the preference information, there may still exist many such value functions. The idea of evaluating all functions that are compatible with the preference information was firstly introduced in UTA GMS method (see [36,37]).

Similarly, in the UTA-based method, we propose that the value that is obtained from a bundle is the sum of the marginal values acquired from each output level in the bundle (we assume preference independence). Moreover the social welfare function is assumed to be sum of the bundle values (i.e. we use a utilitarian framework). These functions are in the following forms:

$$
\begin{aligned}
& \quad B V\left(b_{i}^{k}\right)=\sum_{j=1}^{n} M V_{j}\left(a_{i j}^{k}\right) \text { where } b_{i}^{k}=\left(a_{i 1}^{k}, a_{i 2}^{k}, \ldots, a_{i n}^{k}\right) \\
& \forall i=1,2, \ldots, m, \quad \forall k=1,2, \ldots, N \\
& S W\left(a^{k}\right)=\sum_{i=1}^{m} \sum_{j=1}^{n} M V_{j}\left(a_{i j}^{k}\right) \quad \forall k=1,2, \ldots, N
\end{aligned}
$$

with the normalization constraints below,

$$
M V_{j}\left(a_{j_{*}}\right)=0, \quad \sum_{j=1}^{n} M V_{j}\left(a_{j}^{*}\right)=1, \quad M V_{j}\left(a_{i j}\right) \geq 0
$$

$\forall j=1,2, \ldots, n, \quad \forall i=1,2, \ldots, m$

where $a_{j_{*}}$ and $a_{j}^{*}$ are the least and most preferable levels of output $j$, respectively. Therefore, in this approach inferring the marginal

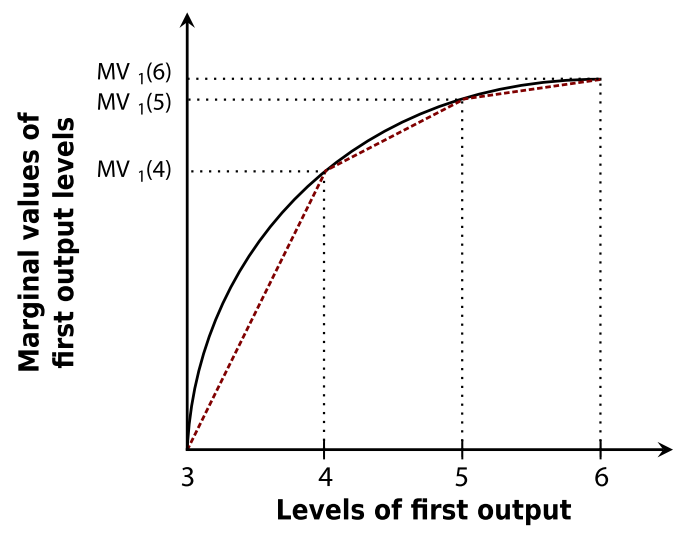

Fig. 1. Concave marginal value function and its piecewise linear approximation.

value functions of the outputs will be sufficient to calculate social welfare scores of the alternatives.

There are different UTA applications in the literature, each with its own assumptions on the shape of the marginal value functions. They can be linear $[38,39]$, piecewise linear $[32,40,41]$, or monotone [42]. In our problem setting, we assume that all $M V_{j}(.) \mathrm{s}$ are concave and approximate them in our mathematical models by piecewise linear approximation. This assumption is used to (partially) reflect the fairness concerns of the DM into the model. When concave marginal value functions are used, the total value increases (the total value that the alternative brings becomes higher) as the levels of an output distributed to users get closer, everything else being the same.

Fig. 1 illustrates a concave marginal value function for an output with four different levels and the corresponding piecewise linear function.

We now discuss our interactive UTA-based algorithm, which finds the best alternative (or a small subset of most preferred ones) using basic UTA principles. (See [43] for an alternative methodology based on robust ordinal regression to find the best alternative in an efficient way for general MCDM settings.)

At each iteration, preference information of the DM is gathered by asking bundle comparison questions. Then the algorithm eliminates an alternative if there exists any other alternative that brings higher social welfare value for all possible marginal value assignments that are compatible with given preference information. This loop is repeated until the number of remaining alternatives is less than or equal to a predetermined threshold value $K$.

\section{Algorithm 1.}

Step 1: Initialization. Set REMAIN $=\left\{a^{1}, a^{2}, \ldots, a^{N}\right\}$. Find the emdominated alternatives and remove them from the set REMAIN.

Step 2: UTA eliminations. For all pairs of alternatives, make pairwise comparisons using the Comparison subroutine. Make the necessary eliminations and update set REMAIN accordingly. (Note that no preference information has been incorporated yet.)

Step 3: Take new preference information from the DM by using Vectorpreferenceinfo subroutine. Add the information as a constraint to the UTA-based model and go to Step 4 .

Step 4: Preference Information Eliminations. Take the first pair that is not compared yet in set REMAIN and call the Comparison 
Table 2

Elimination rules for UTA-based algorithm.

\begin{tabular}{lll}
\hline Is $S W\left(a^{k}\right) \geq S W\left(a^{k^{\prime}}\right)$ & Is $S W\left(a^{k^{\prime}}\right) \geq S W\left(a^{k}\right)$ & Results \\
possible? & possible? & \\
\hline Yes & Yes & Inconclusive \\
Yes & No & Eliminate alternative $a^{k^{\prime}}$ \\
No & Yes & Eliminate alternative $a^{k}$ \\
\hline
\end{tabular}

subroutine. Make the necessary eliminations and update set REMAIN accordingly. If all pairs in REMAIN have not been checked yet, repeat this step. Otherwise, if the number of alternatives in set REMAIN is higher than $K$, go to step 3. If not, go to Step 5.

Step 5: Stop and report REMAIN.

Let us now explain Vectorpreferenceinfo and Comparison subroutines in more detail.

\section{Vectorpreferenceinfo}

This subroutine is used to select the vectors to be asked to the DM. We tried three alternative methods to choose these vectors, which are based on random selection, distance to an ideal vector and pairwise distances between the set of vectors.

Random selection: We randomly choose two vectors.

Ideal: We create an ideal vector $\left(\left(\right.\right.$ ideal $_{1}$, ideal $_{2}, \ldots$, ideal $\left._{n}\right)$ where $i_{\text {deal }}=\max _{\forall k \in A, \forall i \in I} a_{i j}^{k}$ ) and calculate the Euclidean distances between the ideal vector and each vector (bundle) in the given set of alternatives. The vectors to be asked are chosen starting from the ones which have smallest distance to the ideal vector. This method aims to gather effective preference information from the DM by asking her to compare strong candidates.

Minimum pairwise distance: We calculate the Euclidean distances between all pairs of vectors in the given set of alternatives. We choose the vectors that have the minimum distance to each other. This method aims to gather effective preference information from the DM by asking her to compare candidates that are more difficult to distinguish.

We also tried a maximum pairwise distance strategy so as to ensure that the chosen vectors have enough diversity (rather than high similarity). However, the computational results demonstrated that this method is outperformed by the others. The poor performance of this strategy may be due to the fact that such comparisons are ineffective in reducing the set of compatible value assignments.

\section{Comparison}

This subroutine is used to compare alternatives with each other. For any two alternatives $a^{k}$ and $a^{k^{\prime}}$ and given preference information, we check if $S W\left(a^{k}\right)$ can be higher than $S W\left(a^{k^{\prime}}\right)$ using UTAbased model. Then we check if $\operatorname{SW}\left(a^{k^{\prime}}\right)$ can be higher than $\operatorname{SW}\left(a^{k}\right)$. We make the necessary eliminations using Table 2 provided below.

Given preference information, if $\operatorname{SW}\left(a^{k}\right) \geq \operatorname{SW}\left(a^{k^{\prime}}\right)\left(\operatorname{SW}\left(a^{k^{\prime}}\right) \geq\right.$ $\left.S W\left(a^{k}\right)\right)$ is possible but $S W\left(a^{k^{\prime}}\right) \geq S W\left(a^{k}\right)\left(S W\left(a^{k}\right) \geq S W\left(a^{k^{\prime}}\right)\right)$ is not possible, then we conclude that the alternative $k\left(k^{\prime}\right)$ is better. If both cases are possible we do not make any eliminations.

Let us now review the steps of the Algorithm 1 for the provided example (Example 2) where the DM tries to find the best alternative among six alternatives.

Step 1 . Checks the em-dominance relation among the alternatives. It eliminates $a^{3}$ since $a^{4}$ em-dominates $a^{3}$.

Step 2. Compares the alternatives just considering the assumptions on MVFs (being increasing and concave) without obtaining any preference information. At this step $a^{5}$ is eliminated (the total levels of both outputs that are distributed by $a^{2}$ and $a^{5}$ are the same and $a^{2}$ distributes first output more equally than $a^{5}$ ). The same relation is observed between $a^{4}$ and $a^{6}$, hence $a^{6}$ is also eliminated.
Step 3. The DM is asked to compare bundles $(4,6)$ and $(5,5)$ (assume that the question selection method is based on the distance from the ideal vector). Assume that the DM chooses $(5,5)$. This preference information is added to UTA-based model described below as a constraint (constraint (7)).

Step 4. Through solving the related mathematical models, we conclude that $a^{1}$ cannot be better than $a^{4}$, hence it is eliminated. The remaining alternatives are $a^{2}$ and $a^{4}$.

Step 3. The DM is asked to compare bundles $(8,2)$ and $(3,7)$. This preference information is added to UTA-based model as a constraint (constraint (7)).

Step 4 . Through solving the related mathematical models, $a^{2}$ is eliminated.

Step 5. The algorithm returns $a^{4}$ as the solution.

UTA-based model introduced below checks if alternative $a^{k}$ can have higher social welfare value than alternative $a^{k^{\prime}}$ considering the DM's preference information. Note that this is a feasibility model.

\section{UTA-based model}

Sets:
I: $\quad$ the set of users $\{1, \ldots, m\}$.
$J: \quad$ the set of outputs $\{1, \ldots, n\}$.
$Q$ : the pairwise comparison information gathered so far
$\left\{\left(p, p^{\prime}\right): p\right.$ is preferred over $\left.p^{\prime} \& p, p^{\prime} \in \mathbb{R}^{n}\right\}$.
$C_{j}$ : the vector that stores unique values of output $j$ in an increasingly ordered manner.

\section{Parameters:}

$L_{j}: \quad$ the number of different levels in output $j$.

$T_{i j k}$ : The rank of $a_{i j}^{k}$ in set $C_{j}$ where $i \in I, j \in J$ and $a^{k} \in A$.

$T_{p j}$ : The rank of $p_{j}$ in set $C_{j}$ where $p \in Q, j \in J$.

$\epsilon: \quad$ a small positive number to ensure the MVFs are increasing.

$\gamma$ : a small positive number to ensure the MVFs are strictly concave.

$\Omega$ : $\quad$ a small positive number to incorporate strict preference information.

\section{Variables:}

$M V_{j t}: \quad$ the value of the $t$ th minimum level in output $j$.

$B V_{p}: \quad$ the total value achieved from the bundle $p$ where $p=\left\{p_{1}, \ldots, p_{n}\right\}$ and $p \in Q$.

$S W_{k}$ : the total social welfare that alternative $k$ brings where $k \in A$.

maximize 0

subject to $M V_{j, t+1}-M V_{j t} \geq \epsilon \quad \forall j \in J, t \in\left\{1, \ldots, L_{j}-1\right\}$

$4 \frac{M V_{j, t+1}-M V_{j t}}{C_{j, t+1}-C_{j t}}-\frac{M V_{j, t+2}-M V_{j, t+1}}{C_{j, t+2}-C_{j, t+1}} \geq \gamma$

$\forall j \in J, t \in\left\{1, \ldots, L_{j}-2\right\}$

$\sum_{j \in J} M V_{j L_{j}}=1$

$M V_{j 1}=0 \quad \forall j \in J$

$B V_{p}=\sum_{j \in J} M V_{j T_{p j}} \quad \forall p \in Q$

$B V_{p}-B V_{p^{\prime}} \geq \Omega \quad \forall\left(p, p^{\prime}\right) \in Q$

$S W_{k}=\sum_{j \in J, i \in I} M V_{j T_{i j k}}$ 
$S W_{k^{\prime}}=\sum_{j \in J, i \in I} M V_{j T_{i j k^{\prime}}}$

$S W_{k}-S W_{k^{\prime}} \geq 0$

$M V_{j t} \geq 0 \quad \forall j \in J, t \in\left\{1, \ldots, L_{j}\right\}$

The model tries to assign values to outputs in such a way that social value score of alternative $k$ will be greater than social value score of alternative $k^{\prime}$. Constraint sets (2) and (3) ensure that the marginal value functions will be increasing and concave, respectively. Parameter $\gamma$ determines the concavity levels of the marginal value functions. Constraint sets (4) and (5) are for normalization and guarantee that the social welfare values of all alternatives are in the range $[0-m]$. Constraint set (6) assigns a BV score to each bundle $p$ in the preference information set. Constraint set (7) incorporates the provided information by the DM to into the model. Constraint sets (8) and (9) assign social welfare values to alternatives $k$ and $k^{\prime}$, respectively. Constraint (10) checks if alternative $a^{k}$ can bring higher social welfare value than alternative $a^{k^{\prime}}$.

UTA-based approach introduces concave marginal value functions hence encourages a more equitable distribution for each of the outputs over users regardless of the levels of other outputs that they receive. That is, using additivity over users, we make this underlying assumption that the outputs are not substitutable and hence a more equitable distribution is always desired regardless of users' positions with respect to the other outputs.

Let us consider the following two alternatives in Example 2: $a^{2}=\left(\begin{array}{ll}5 & 5 \\ 6 & 2\end{array}\right)$ and $a^{5}=\left(\begin{array}{ll}3 & 5 \\ 8 & 2\end{array}\right)$. The first output is distributed in a more equitable manner in $a^{2}$ but this occurs at the cost of making second user, who was worse off with respect to second output, have less of first output compared to $a^{5}$. In UTA-based approach, $a^{2}$ is considered better since, everything else being the same, the first output is distributed in a more equitable manner. However, one can argue that redistribution is only meaningful and social welfare increasing when one user is definitely underprivileged and redistribution alleviates this underprivilege, which is not the case in this example. In such cases, the UTA-based approaches will not be of use and the preference model of a DM who would prefer e.g. $\left(\begin{array}{ll}3 & 5 \\ 8 & 2\end{array}\right)$ over $\left(\begin{array}{ll}5 & 5 \\ 6 & 2\end{array}\right)$ on the grounds that the outputs may be substitutable cannot be taken into account. This is due to the additivity assumption of the UTA-based approach.

There exists a large body of work in the economics literature discussing inequality in single good distributions like income. When a single good is distributed, most of the literature agrees on the suitability of using nonadditive social welfare functions rather than assuming separability [44-46]. The convex cone based approach, which we discuss now, alleviates some drawbacks of the UTA-based approach as it (partially) relaxes the additivity assumption for the social welfare function used. That is, the convex cones approach will allow a DM to prefer the first distribution over the second in the above example as we will elaborate in the next section. (The interested reader is referred to [47], which discusses another possible non additive approach within the UTA family methods.)

\subsection{Convex cone based approach}

In this section, we discuss the convex cone based approach, which is widely used in the MCDM literature [48-52]. Convex cones are used in MCDM problems to incorporate preference information in the model. This method assumes that the underlying value function of the DM is quasi-concave and is based on

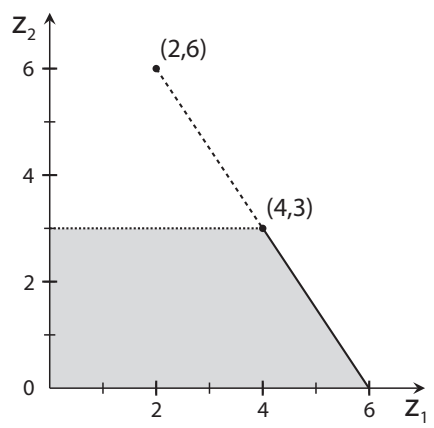

Fig. 2. $C((2,6) ;(3,4))$ and cone dominated region.

eliminating the alternatives that are inferior to the cones generated based on preference information that she provides [49]. We first give the main definitions and results used in the classical MCDM choice problems, where alternatives are vectors. We then discuss an extension of the approach to cases where each alternative shows the allocation of a single output over multiple users and the DM has an equitable preference model (discussed in [53]). Finally, we provide the extension we suggest for problems where the alternatives are defined as matrices.

Definition 4. Given a set of $\mathrm{k}$ vectors, such that $z^{1}, \ldots, z^{k} \in \mathbb{R}^{m}$, the cone $C\left(z^{1}, \ldots, z^{k-1} ; z^{k}\right)$ is defined, where $z^{\ell}: \ell \neq k$ are the upper generators and $z^{k}$ is the lower generator as follows: $C\left(z^{1}, \ldots, z^{k-1} ; z^{k}\right)=\left\{z \mid z=z^{k}+\sum_{\ell \neq k} \mu_{\ell}\left(z^{k}-z^{\ell}\right), \mu_{\ell} \geq 0\right\}$. The cone dominated region of $C\left(z^{1}, \ldots, z^{k-1} ; z^{k}\right)$ is denoted by $C D\left(z^{1}, \ldots, z^{k-1} ; z^{k}\right)$ and defined as follows $C D\left(z^{1}, \ldots, z^{k-1} ; z^{k}\right)=$ $\left\{z^{\prime} \mid z^{\prime} \leq z\right.$ where $\left.z \in C\left(z^{1}, \ldots, z^{k-1} ; z^{k}\right)\right\}$.

If the value function of the DM (SWF) is quasi-concave, the following holds [49],

Lemma 1. For any $z^{c} \in C\left(z^{1}, \ldots, z^{k-1} ; z^{k}\right), S W\left(z^{c}\right) \leq S W\left(z^{k}\right)$. Also, for any $z^{\prime} \in C D\left(z^{1}, \ldots, z^{k-1} ; z^{k}\right), S W\left(z^{\prime}\right) \leq S W\left(z^{k}\right)$.

Each point $z^{\prime} \in C D\left(z^{1}, \ldots, z^{k-1} ; z^{k}\right)$ is called cone dominated.

To illustrate, suppose that we have $(2,6)$ and $(4,3)$ as alternatives and the DM prefers $(2,6)$ over $(4,3)$. Fig. 2 shows the 2-point cone generated by these alternatives. The solid line represents $C((2$, $6) ;(4,3))$ and the gray area is the cone-dominated region, $C D((2$, $6) ;(4,3))$. Any alternative in this region is cone dominated.

Linear programming models can be used to check if an alternative is in the cone dominated region. For an alternative $z^{I}$, the following feasibility model checks if $z^{I}$ is in the cone dominated region $C D\left(z^{1}, \ldots, z^{k-1} ; z^{k}\right)$. The right hand side of constraint set (13) corresponds to a point on $C\left(z^{1}, \ldots, z^{k-1} ; z^{k}\right)$, which (vector) dominates $z^{I}$. If this model is feasible, $z^{I} \in C D\left(z^{1}, \ldots, z^{k-1} ; z^{k}\right)$.

minimize 0

subject to $z_{i}^{I} \leq z_{i}^{k}+\sum_{\ell=1}^{k-1} \mu_{\ell}\left(z_{i}^{k}-z_{i}^{\ell}\right), \quad$ for $i=1, \ldots, m$

$\mu_{\ell} \geq 0, \quad$ for $\ell=1, \ldots, k-1$

A large body of the literature using convex cones in MCDM problems do not touch upon the concept of equitability. Karsu et al. [53] extend the use of convex cones for allocation settings where a single output is distributed to multiple users and impartiality holds. Since the preference model of the DM is assumed to be equitable, impartiality holds, which implies that the value function of the DM is symmetric quasi-concave. This assumption 

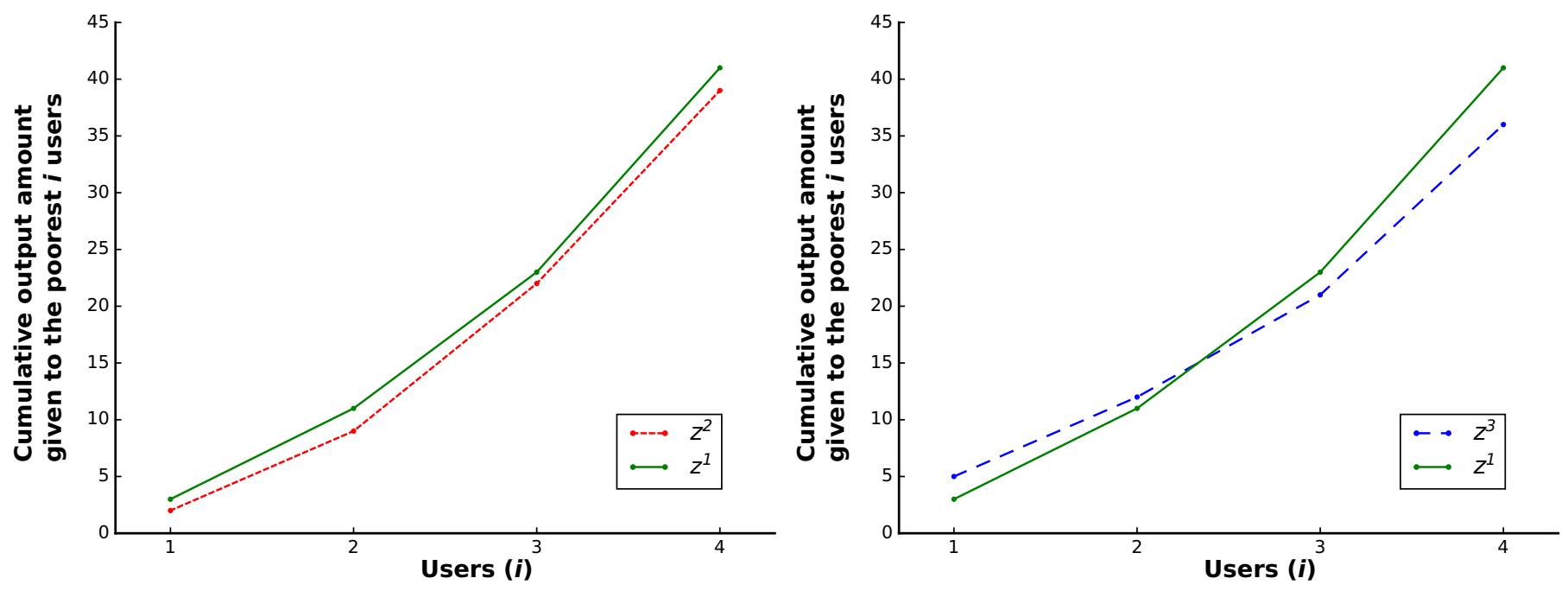

Fig. 3. Generalized Lorenz dominance illustration.

implies that each vector (allocation) of size $m$ will have $m$ ! permutations and the DM is indifferent to all these permutations. Hence given single pairwise preference information, one can generate multiple cones considering various permutations of the upper and lower generators. Let us re-consider the example described above. If the DM prefers $(2,6)$ over $(4,3)$, impartiality implies that the DM prefers any permutation of $(6,2)$ over any permutation of $(4,3)$. So in addition to $C((2,6) ;(4,3))$ we can generate the cones $C((2,6) ;(3,4)), C((6,2) ;(3,4))$ and $C((6,2) ;(4,3))$ and eliminate the alternatives which are inferior to any of these cones.

Considering multiple permutation cones increases the amount of inference one can make from the preference information. However, note that, when one has a cone generated by $n$ vectors of size $m$, the number of permutation cones to be considered becomes $m !^{n}$. Karsu et al. [53] introduce results to handle this complexity. The study also involves using a different dominance relation than the vector dominance relation, namely the generalized Lorenz dominance (also called equitable dominance) relation, which is defined below.

Definition 5. Let $z^{k}$ denote the permutation of $z^{k}$ such that $z^{k}$ : $\vec{z}_{1}^{k} \leq \vec{z}_{2}^{k} \leq \ldots \leq \vec{z}_{m}^{k}$ where $m$ is the number of users. $\vec{z}^{k}$ is called the ordered vector of $z^{k}$. Let $\bar{Q}\left(z^{k}\right)$ denote the cumulative ordered vector of $z^{k}$ defined as follows:

$\bar{Q}\left(z^{k}\right)=\left(\bar{Q}_{1}\left(z^{k}\right), \bar{Q}_{2}\left(z^{k}\right), \ldots, \bar{Q}_{m}\left(z^{k}\right)\right) \quad$ where $\quad \bar{Q}_{i}\left(z^{k}\right)=\sum_{t=1}^{i} \bar{z}^{k}$ $\forall i \in I, \quad I=\{1,2, \ldots, m\}$.

That is, $\bar{Q}_{i}\left(z^{k}\right)$ shows the total output amount provided to the poorest $i$ users in the distribution.

Theorem 1. Given two alternatives $z^{1}, z^{2} \in \mathbb{R}^{m}$,

$$
z^{1} \preceq_{G L} z^{2} \quad\left(z^{2} \quad \text { generalized Lorenz dominates } \quad z^{1}\right) \Longleftrightarrow \bar{Q}_{i}\left(z^{1}\right) \leq
$$
$\bar{Q}_{i}\left(z^{2}\right) \forall i \in I[10]$.

Generalized Lorenz dominance is introduced as an extension of the widely-known Lorenz dominance concept used in the economics literature [27]. It can be used to compare distribution vectors over anonymous users even when the means of the distributions are not equal. Moreover, pairs of alternatives for which vector dominance remains inconclusive, could be compared using generalized Lorenz dominance. For example, assume that we have three alternatives where $z^{1}=(12,7,3,18), z^{2}=(2,7,12,18)$, and $z^{3}=(9,7$, $15,5)$. None of the vectors is dominated in the vector dominance sense. However, since $\bar{Q}\left(z^{1}\right)=(3,10,22,40)$ and $\bar{Q}\left(z^{2}\right)=(2,9,21$, $39), z^{2} \preceq_{G L} z^{1}$. Fig. 3 shows the generalized Lorenz curves of the alternatives provided. It is seen that the cumulative output amount given to the poorest $i$ users in $z^{1}$ is always higher than that of $z^{2}$; hence the generalized Lorenz curve of $z^{1}$ is always above that of $z^{2}$. However, there is no dominance between $z^{3}$ and $z^{1}$ since the two curves intersect.

When dealing with single benefit distributions, Karsu et al. [53] eliminate an alternative if it is generalized Lorenz dominated by any of the permutation cones. It is proved that, rather than considering all the permutation cones, it is sufficient to use the cone generated by the ordered versions of the generators. In order to check if an alternative $z^{I}$ is (generalized Lorenz) dominated by any of the permutation cones the following model is solved [53]:

$\operatorname{maximize} \sum_{h=1}^{m} h r_{h}-\sum_{h=1}^{m} \sum_{i=1}^{m} d_{h i}$

subject to $z_{i}^{c}-\sum_{\ell=1}^{k-1} \mu_{\ell}\left({z_{i}}^{k}-{z_{i}}^{\ell}\right)={z_{i}}^{k} \quad$ for $i=1, \ldots, m$

$r_{h}-d_{h i}-z_{i}^{c} \leq 0 \quad$ for $i, h=1, \ldots, m$

$\sum_{j=1}^{k} \vec{z}_{j}^{I} \leq h r_{h}-\sum_{i=1}^{m} d_{h i} \quad$ for $h=1, \ldots, m$

$d_{h i} \geq 0 \quad$ for $i, h=1, \ldots, m$

$\mu_{\ell} \geq 0 \quad$ for $\ell=1, \ldots, k-1$

where $r_{h}$ and $d_{h i}$ are auxiliary variables used to ensure that cumulative ordered vector of $z^{c}$ is found (at optimality, $h r_{h}^{*}-\sum_{i=1}^{m} d_{h i}^{*}=$ $\bar{Q}_{h}\left(z^{c}\right)$. Note that the model has alternate optima, $r_{h}^{*}=\bar{z}_{h}^{c}+g$, where $g$ is a scalar and $d_{h i}^{*}=0$ for $i: z_{i}^{c}>z_{h}^{c}$ and $d_{h i}^{*}=z_{h}^{c}-z_{i}^{c}+g$ for $i: z_{i}^{c} \leq z_{h}^{c}$. These ensure that at optimality the difference term $\left.h r_{h}^{*}-\sum_{i=1}^{m} d_{h i}^{*}=\bar{Q}_{h}\left(z^{c}\right)\right)$ [54]. This model checks if there exist $z^{c} \in$ $C\left(z^{1}, \ldots, z^{k-1} ; z^{k}\right)$ such that $\bar{Q}\left(z^{I}\right) \leq \bar{Q}\left(z^{c}\right)$. Constraint set (16) creates $z^{c}$ such that $z^{c} \in C\left(z^{1}, \ldots, z^{k-1} ; z^{k}\right)$. Constraint set (17) together with the objective function ensures that at optimality, $h r_{h}^{*}-$ $\sum_{i=1}^{m} d_{h i}^{*}=\bar{Q}_{h}\left(z^{c}\right)$ and constraint set (18) guarantees that $\bar{Q}\left(z^{I}\right) \leq$ $\bar{Q}\left(z^{c}\right)$. 
Karsu et al. [53] consider ranking problems where alternatives are allocation vectors of a single output to multiple users. We suggest a further extension of the convex cone method to problems where the alternatives are defined as matrices. We assume that social welfare is a symmetric quasi-concave function of the bundle values, which are assumed to be additive.

We assume the DM has an equitable preference model over the distribution vector of these bundle values, hence use the convex cones method discussed in [53] (with the generalized Lorenz dominance relation). The bundle values are calculated as the weighted sum of the scaled output levels. The scaled matrix $a^{k^{s}}$ for an alternative $a^{k}$ is generated as follows: $a_{i j}^{k^{s}}=\left(a_{i j}^{k}-\right.$ $\left.\min _{i \in I, k \in A} a_{i j}^{k}\right) /\left(\max _{i \in I, k \in A} a_{i j}^{k}-\min _{i \in I, k \in A} a_{i j}^{k}\right)$. For the sake of simplicity, from now on we use $a^{k}$ for the scaled levels, too. $B V\left(b_{i}^{k}\right)$ is calculated as $B V\left(b_{i}^{k}\right)=\sum_{j \in J}\left(w_{j} a_{i j}^{k}\right)$.

Then, the previous model becomes,

$\operatorname{maximize} \sum_{h=1}^{m} h r_{h}-\sum_{h=1}^{m} \sum_{i=1}^{m} d_{h i}$

subject to $z_{i}^{c}-\sum_{\ell=1}^{k-1} \mu_{\ell}\left(\overrightarrow{\left(w a^{k}\right)_{i}}-\overrightarrow{\left(w a^{\ell}\right)_{i}}\right)=\overrightarrow{\left(w a^{k}\right)_{i}}$ for $i=1, \ldots, m$

$r_{h}-d_{h i}-z_{i}^{c} \leq 0 \quad$ for $i, h=1, \ldots, m$

$\sum_{j=1}^{h} \overrightarrow{\left(w a^{I}\right)_{j}} \leq h r_{h}-\sum_{i=1}^{m} d_{h i} \quad$ for $h=1, \ldots, m$

$\sum_{j=1}^{n} w_{j}=1$

$d_{h i} \geq 0 \quad$ for $i, h=1, \ldots, m$

$w_{j} \geq 0 \quad j=1, \ldots, n$

$\mu_{\ell} \geq 0 \quad$ for $\ell=1, \ldots, k-1$

This model checks if there exists any $z^{c}$ vector on $C\left(w a^{1}, w a^{2}, \ldots, w a^{k-1} ; w a^{k}\right)$, that generalized Lorenz dominates a given alternative $a^{I}\left(w a^{I}\right)$ for any weight value $(w)$. Since the weight vectors are also unknown, the model discussed above is non-linear. Moreover, even when the above model is feasible we cannot eliminate an alternative, since it could have been cone dominated for some $w$ vector and not dominated for others. To be affirmative, one should ensure that alternative $a^{I}$ is cone dominated over the entire feasible weight space. In order to handle this non-linearity and be conclusive, we use discretization and perform a parametric search over the entire (discretized) feasible weight region.

\subsubsection{The convex cones algorithm}

We now describe the convex cone-based algorithm we use for our problem setting. We will explain the algorithm for problems with two outputs and for the case where only 2-point cones (these are cones with only two generators) are used. It is straightforward to generalize the algorithm for problems with more than two outputs with an appropriate discretization of the feasible weight space. The algorithm can easily be modified if one wants to use $k$-point cones (cones with $k-1$ upper generators and one lower generator). We assume that there are $N$ alternatives and $m$ users as before. In addition to the set REMAIN, which keeps the alternatives not eliminated so far, we define the following sets: the set CONES stores all the alternative pairs on which the DM provides preference information. The set POSW1 stores the possible weight values for the first output, which are compatible with the preference information that the DM provided. Recall that we discretize the weight space.

\section{Algorithm 2.}

Step 1: Initialization. CONES $=\emptyset$. REMAIN $=\left\{a^{1}, a^{2}, \ldots, a^{N}\right\}$. Find the em-dominated alternatives and remove them from REMAIN. POSW1 $=\{0,0.05,0.1,0.15, \ldots, 0.95,1\}$.

Step 2: Take new preference information from the DM using Holisticpreferenceinfo subroutine and let $a^{U}$ and $a^{L}$ indicate preferred and not-preferred alternatives, respectively. Remove $a^{L}$ from REMAIN. If the number of alternatives in the set REMAIN is greater than $K$, narrow the possible weight interval by using Narrowweight subroutine (if possible) and go to Step 3. Otherwise, STOP.

Step 3: Update CONES $=\{C O N E S\} \cup\left(a^{U} ; a^{L}\right)$ and remove the cone dominated alternatives from REMAIN by using Conedominancecheck subroutine. If the number of alternatives in the set REMAIN is greater than $K$, go to Step 2. Otherwise, STOP.

Let us now explain each subroutine in more detail.

\section{Holisticpreferenceinfo}

This subroutine is used to determine the alternatives to ask the DM for pairwise comparison. It creates an ideal alternative, IDEAL, such that $I D E A L_{i j}=\max _{\forall k \in A, \forall i \in I} a_{i j}^{k}$ and calculates the Euclidean distance between each alternative in set REMAIN and IDEAL. Then the DM is asked to choose between two alternatives that have the minimum distances.

\section{Narrowweight}

This subroutine is used to narrow the possible weight interval of the first output in line with the preference information. Suppose that the DM is asked to choose between two alternatives in $\mathbb{R}^{(m \times n)}$. Let $a^{U}$ be the preferred alternative and $a^{L}$ be the alternative which is not preferred. We eliminate the weights that satisfy the following inequality $\bar{Q}\left(a^{U}\left[\begin{array}{c}w_{1} \\ \ldots \\ w_{n}\end{array}\right]\right) \leq \bar{Q}\left(a^{L}\left[\begin{array}{c}w_{1} \\ \ldots \\ w_{n}\end{array}\right]\right)$ based on Remark 1.

Remark 1. If the DM prefers $a^{U}$ over $a^{L}$, then $a^{L}$ cannot generalized Lorenz dominate $a^{U}$. Then, from the definition of generalized Lorenz dominance, we are sure that the following inequality $\bar{Q}\left(a^{U}\left[\begin{array}{l}w_{1} \\ \ldots \\ w_{n}\end{array}\right]\right) \leq \bar{Q}\left(a^{L}\left[\begin{array}{l}w_{1} \\ \ldots \\ w_{n}\end{array}\right]\right)$ cannot hold. The weight values that satisfy the above inequality should be eliminated as they would lead to a less preferred alternative to generalized Lorenz dominate a more preferred one, contradicting with the assumptions made on the preference model.

\section{Conedominancecheck}

This subroutine is used to find the cone dominated alternatives in the set REMAIN. The subroutine checks if $a^{I}\left(w a^{I}\right)$ is cone dominated by any $C\left(w a^{U^{\prime}} ; w a^{L^{\prime}}\right)$ such that $\left(a^{U^{\prime}}, a^{L^{\prime}}\right) \in$ CONES $\forall w \in P O S W 1$ where $a^{I} \in$ REMAIN. To eliminate an alternative, it is sufficient to ensure that for any weight level possible, there exists a cone dominating the alternative. If so, that alternative is removed from the set REMAIN. This is repeated for all the alternatives in the set REMAIN.

Let us now review the steps of the Algorithm 2 for the provided example (Example 2) where the DM tries to find the best alternative among six alternatives. We assume that the underlying social welfare function of the DM is $S W\left(a^{k}\right)=\left(0.7 a_{11}^{k}+0.3 a_{12}^{k}\right)\left(0.7 a_{21}^{k}+\right.$ $\left.0.3 a_{22}^{k}\right)$. 
Step 1. Checks the em-dominance relation among the alternatives. It eliminates $a^{3}$ since $a^{4}$ em-dominates $a^{3}$.

Step 2. The DM is asked to compare $a^{2}$ and $a^{4}$ and prefers $a^{2}$ to $a^{4}$. This preference information eliminates $a^{4}$ and narrows the possible weight interval for the first output to [0.7-1].

Step 3. $\mathrm{C}\left(w a^{2} ; w a^{4}\right)$ is generated for all the possible discretized weights $(0.7,0.75,0.8,0.85,0.9,0.95,1)$. The remaining alternatives $\left(a^{1}, a^{5}, a^{6}\right)$ are checked if any of them is dominated using the Cone-based model (described below). In this example, none of the alternatives are dominated so no elimination can be made.

Step 2. The DM is asked to compare $a^{2}$ and $a^{6}$ and prefers $a^{2}$ to $a^{6}$. This information eliminates $a^{6}$ but does not narrow the possible weight interval any further.

Step 3. Cones $C\left(w a^{2} ; w a^{6}\right)$ are generated and the remaining alternatives $\left(a^{1}, a^{5}\right)$ are checked for cone dominance using Conebased model. $a^{1}$ is dominated by $\mathrm{C}\left(w a^{2} ; w a^{6}\right)$ for all $w$, hence it is eliminated.

Step 2. The DM is asked to compare $a^{2}$ and $a^{5}$ and prefers $a^{2}$ to $a^{5}$. STOP. The algorithm returns $a^{2}$.

Cone-based model introduced below checks if an alternative is in the cone dominated region for a given weight vector. Suppose we want to check if $a^{I}$ is in the cone dominated region of the cone generated by the alternatives $a^{U}$ and $a^{L}$. Remember that our alternatives are represented by matrices. We first calculate the BV vectors for the alternatives by using weighted sum of their output levels. After we obtain bundle value vectors for the alternatives, we use Cone-based model to check if $a^{I}$ is cone dominated.

\section{Cone-based model}

Assume that the DM is asked to choose between two alternatives and $a^{U}$ represents the alternative that the DM prefers and $a^{L}$ represents the alternative that the DM does not prefer. The following model checks if alternative $a^{I}$ is in the cone dominated region generated by $a^{U}$ and $a^{L}$.

Parameters:

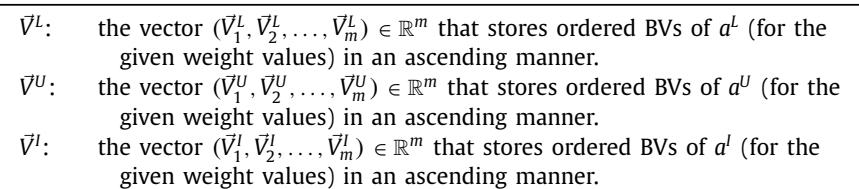

\begin{tabular}{ll}
\hline Variables: \\
\hline$\mu: \quad$ the scalar for $\mathrm{C}\left(\vec{V}^{U} ; \vec{V}^{L}\right)$. \\
$V^{c}: \quad$ a vector $\in \mathbb{R}^{m}: V^{c} \in \mathrm{C}\left(\vec{V}^{U} ; \vec{V}^{L}\right)$. \\
$r_{h}: \quad$ auxiliary variables used to ensure that the cumulative ordered vector \\
$\quad$ of $V^{c}$ is found. \\
$\quad \begin{array}{l}\text { auxiliary variables used to ensure that the cumulative ordered vector } \\
\quad \text { of } V^{c} \text { is found. }\end{array}$ \\
\end{tabular}

$\operatorname{minimize} \sum_{h=1}^{m} h r_{h}-\sum_{h=1}^{m} \sum_{i=1}^{m} d_{h i}$

subject to $V_{i}^{c}-\mu\left(\vec{V}_{i}^{L}-\vec{V}_{i}^{U}\right)=\vec{V}_{i}^{L} \quad$ for $i=1, \ldots, m$

$r_{h}-d_{h i}-V_{i}^{c} \leq 0 \quad$ for $i, h=1, \ldots, m$

$h r_{h}-\sum_{i=1}^{m} d_{h i} \geq \sum_{j=1}^{h} \vec{V}_{j}^{I} \quad$ for $h=1, \ldots, m$

$d_{h i} \geq 0 \quad$ for $i, h=1, \ldots, m$

$\mu \geq 0$
Constraint set (30) creates a $V^{c}$ vector in $C\left(V^{U} ; V^{L}\right)$. Constraint sets (31) and (32) ensure that the created $V^{c}$ generalized Lorenz dominates $V^{I}$ by using $r_{h}$ and $d_{h i}$ auxiliary variables. Constraints (33) and (34) are non-negativity constraints.

As discussed before, assuming a symmetric quasi-concave social welfare function partially handles the issue of preferential independence by relaxing the additivity assumption of the UTA-based approach. To elaborate, recall the previous example consisting of alternatives $a^{2}=\left(\begin{array}{ll}5 & 5 \\ 6 & 2\end{array}\right)$ and $a^{5}=\left(\begin{array}{ll}3 & 5 \\ 8 & 2\end{array}\right)$, in which the UTAbased approach always gives $a^{2}$ more social value. However, there may be symmetric quasi-concave function forms representing different comparisons. Consider the following types of social welfare functions, which are symmetric quasi-concave: additive (social welfare is the sum of bundle values), multiplicative (social welfare is the product of bundle values [53]), Rawlsian (social welfare is the minimum of bundle values [55], see also [56]), and ordered weighted averaging (a rank-based function which gives more weights to worse-off users and returns a weighted sum of the bundle values [57]). For these two alternatives, the bundle value vectors become $(0.5,2 w / 3)$ and $((0.5-2 w / 6), w)$, where $w$ is the weight of the first output. Note that we used scalarized matrices when calculating these utility vectors. An additive function would consider the two options as equally good (as both will have $0.5+2 w / 3$ as the social welfare); however, the results for the other functions would change depending on the weight parameter, allowing more flexibility. For example, when the underlying social welfare function is taken as multiplication of bundle values, the DM would prefer $a^{5}$ over $a^{2}$ when $w=0.2$. The convex cone based approach can take such preferences into account.

\section{Extensions of the UTA-based approach}

In this section we discuss some possible extensions to the UTAbased approach. The first extension offers an alternative way to incorporate the tradeoff between efficiency and equity concerns while the second extension relaxes the assumption that the bundle value functions are the same over all users.

Note that in the UTA-based approach, where the social welfare function is assumed to be an additive function of the marginal values, equity concerns are incorporated via assuming that the MVFs are concave. The parameter $\gamma$ controls the degree of concavity in the MVFs (and hence the degree of inequity aversion in the distribution of outputs). Assuming a concave MVF for inequity aversion is analogous to using a concave utility function for risk aversion in decision making under uncertainty. As $\gamma$ value increases, the inequity-aversion increases, resulting in a potential loss in efficiency. By changing the $\gamma$ parameter value, one can obtain solutions with different levels of efficiency and observe the tradeoff between fairness and efficiency.

An alternative approach would be defining equity measures and incorporating constraints into the models to make sure that any chosen solution respects fairness to some degree. Such an approach would return the efficiency maximizing solution that also satisfies the equity constraints. Note that by an efficiency maximizing solution we mean a social welfare value maximizing solution, where the SWF is utilitarian. In this approach one can use any inequality measure in the constraints (see [8] for a list of measures that can be used). We demonstrate this method using a Rawlsian type inequality measure and ensure that the minimum marginal value enjoyed by a user at any output is larger than a predetermined threshold $\alpha$. Similar to the $\gamma$ parameter in the previous approach, $\alpha$ controls how "equitable" an output distribution to the users should be. One can observe the tradeoff between efficiency and equity by iteratively increasing the threshold. 
Similar to the UTA-based model, the following model checks whether an alternative $k$ can be more efficient than alternative $k^{\prime}$ $\left(S W_{k}-S W_{k^{\prime}} \geq 0\right)$ while satisfying the equity constraints, given preference information.

maximize 0

subject to

constraint sets $2,4,5,6,7,8,9,10,11$

$$
M V_{j T_{i j k}} \geq \alpha \forall i \in I, j \in J
$$

For any pair of not-yet-eliminated alternatives $k$ and $k^{\prime}$, we solve the above model and another version, in which $S W_{k^{\prime}}-S W_{k} \geq$ 0 is ensured in constraint (10) and $M V_{j T_{i j k^{\prime}}} \geq \alpha$ is used in constraint set (37). Then, the elimination rules that are summarized in Table 2 are used. The structure of the overall approach is the same as the UTA-based approach, hence we do not repeat it here. Note that, in this approach we do not assume that the MVFs are concave, but just assume that they are increasing. We call this approach equity index based approach.

We now discuss the second extension of the UTA-based approach. Recall that, in the approaches discussed in Section 3, we consider the bundle values as the decision maker's evaluation of the bundles, hence we assume that they do not depend on the identities of the users. This interpretation is also used in other studies in the literature, especially in settings where a central decision maker makes the allocations $[28,29]$.

The bundle value functions could also be interpreted as functions representing users' subjective satisfaction with the bundles rather a central decision maker's. In such cases, the above assumption may be unrealistic since different users may have different preferences over the bundles.

We have developed an extension of the UTA-based approach, which relaxes the assumption that the bundle value functions are the same over all users and takes user specific marginal value functions. Let $M V_{i j}\left(a_{i j}^{k}\right)$ represent the value derived by user $i$ from the allocation of the $j$ th output in alternative $k$. As in the UTAbased approach discussed in Section 3, the bundle values are calculated as the sum of marginal values and the overall social welfare is the sum of bundle values. In this case, we assume that the preference information is not provided by a central DM, rather by the users themselves. Hence, in the algorithm, pairwise bundle comparisons are requested from each user. The structure of the overall approach is the same as the UTA-based approach: for each pair of alternatives, we check whether one can be better than the other given preference information, by solving a modified version of UTA-based model with user specific marginal values. Since the model is a straightforward extension of the UTA-based model, we do not repeat it here. We then use Table 2 to decide whether an alternative could be eliminated from further consideration. We refer to this algorithm as user-specific UTA based approach and provide the results of our computational experiments in Section 5.

\section{Computational experiments}

We perform computational experiments to check the computational efficiency and the quality of the results for proposed algorithms. We generate problem instances with two outputs $(n=2)$. We use two values for the number of alternatives $(N=25$ and $N=$ $50)$ and two values for the number of users $(m=3$ and $m=5$ ). We create 10 problem instances for each parameter setting. The output levels are randomly generated in the range [10-100]. The algorithms stop when the number of remaining alternatives is less
Table 3

\begin{tabular}{|c|c|c|c|c|c|c|c|}
\hline \multirow[t]{2}{*}{$N$} & \multirow[t]{2}{*}{$m$} & \multirow[t]{2}{*}{ method } & \multicolumn{2}{|c|}{ \# of questions } & \multicolumn{2}{|c|}{ solution time } & \multirow[t]{2}{*}{ accurac } \\
\hline & & & avg. & $\max$ & avg. & $\max$ & \\
\hline \multirow[t]{6}{*}{25} & 3 & Random & 30.4 & 88 & 11.75 & 38.8 & $70 \%$ \\
\hline & & Ideal & 8 & 25 & 5.4 & 14.9 & $80 \%$ \\
\hline & & Min dist & 11.4 & 63 & 5.29 & 20.71 & $70 \%$ \\
\hline & 5 & Random & 29.9 & 99 & 15.9 & 56.28 & $80 \%$ \\
\hline & & Ideal & 4 & 9 & 2.91 & 7.53 & $80 \%$ \\
\hline & & Min dist & 31.6 & 97 & 8.07 & 18.81 & $70 \%$ \\
\hline \multirow[t]{6}{*}{50} & 3 & Random & 8.6 & 35 & 4.18 & 9.6 & $90 \%$ \\
\hline & & Ideal & 1.4 & 3 & 1.23 & 3.41 & $80 \%$ \\
\hline & & Min dist & 2.6 & 5 & 2.42 & 6.05 & $80 \%$ \\
\hline & 5 & Random & 12.6 & 25 & 14.3 & 43.9 & $80 \%$ \\
\hline & & Ideal & 3.77 & 10 & 3.4 & 7.23 & $70 \%$ \\
\hline & & Min dist & 3.8 & 9 & 4.99 & 12.37 & $70 \%$ \\
\hline
\end{tabular}

than or equal to a pre-specified threshold value. In our experiments, we set this threshold value $K$ as $K=0.05 N$.

The algorithms are coded in MATLAB and solved by a dual core (Intel Core i5 $2.40 \mathrm{GHz}$ ) computer with 8GB RAM. All models are solved by CPLEX 12.6 and the solution times are expressed in central processing unit (CPU) seconds.

In these experiments, we set $\gamma=5 \times 10^{-5}, \epsilon=5 \times 10^{-4}$ and $\Omega=$ 0.03 for the UTA-based approach. We assume that the underlying marginal value functions are the square root function of the levels of the outputs, i.e. $M V\left(a_{i j}^{k}\right)=\sqrt{a_{i j}^{k}}$ and simulate the responses of the DM accordingly. Table 3 summarizes the results of our experiments for the UTA-based algorithm. We report the average and maximum values for the number of questions asked and the solution time (in seconds), for each parameter setting and for each question selection strategy (Random, Ideal and Minimum pairwise distance). We also report the accuracy of the results, which is calculated as the percentage of instances in which the actual best alternative is in the set of alternatives returned by the algorithm. Note that this inaccuracy is due to the possible inconsistency between the chosen parameters dictating the functional form $(\gamma, \epsilon)$ and the underlying social welfare function used for simulating the responses.

For the convex cone based method, we assume 5 different underlying social welfare function forms as follows:

1. The sum of bundle values (BVs): $S W_{s}\left(a^{k}\right)=\sum_{i=1}^{m} B V\left(b_{i}^{k}\right)$

2. The multiplication of BVs: $S W_{p}\left(a^{k}\right)=\prod_{i=1}^{m} B V\left(b_{i}^{k}\right)$

3. The minimum of BVs (Rawlsian): $S_{m}\left(a^{k}\right)=\min _{\forall i \in I} B V\left(b_{i}^{k}\right)$

4. Sum of pairwise minima of BVs: $\quad S W_{s p}\left(a^{k}\right)=$ $\sum_{i, i^{\prime} \in I: i \neq i^{\prime}} \min \left(B V\left(b_{i}^{k}\right), B V\left(b_{i^{\prime}}^{k}\right)\right)$.

5. Ordered Weighted Average (OWA) of BVs: $S W_{o}\left(a^{k}\right)=$ $\sum_{i=1}^{m} w_{i}^{\text {owa }} \vec{B}_{i}^{k}$, where $B^{k}$ is the vector of bundle values of $a^{k}$ and $w^{o w a} \in \mathbb{R}^{m}$ is a nonincreasing weight vector.

For the OWA function we take $w^{\text {owa }}=(0.5,0.3,0.2)$ for $m=$ 3 and $w^{o w a}=(0.4,0.3,0.2,0.06,0.04)$ when $m=5$. In all these settings, $a^{k}$ corresponds to the scaled matrix obtained by scalarizing the elements of the original matrix. Recall that in this approach, BVs are calculated as $B V\left(b_{i}^{k}\right)=\left(w_{1} a_{i 1}^{k}\right)+\left(1-w_{1}\right) a_{i 2}^{k}$. We assume 3 different underlying weights for the outputs: $w^{1}=$ $(0.15,0.85), w^{2}=(0.5,0.5)$, and $w^{3}=(0.85,0.15)$.

Tables 4 and 5 summarize the results of our experiments for the convex cone based algorithm. We report the average and maximum values for the number of questions asked and the solution time (in seconds), for each parameter setting. We also report the average and maximum reduction (as percentage) in the weight interval for the first output. Recall that in the convex cone based approach, we narrow down the possible weight interval of the bundle 
Table 4

Convex cone results for additive, Rawlsian, and multiplicative social welfare functions.

\begin{tabular}{|c|c|c|c|c|c|c|c|c|c|}
\hline \multirow[t]{2}{*}{ SWF form } & \multirow[t]{2}{*}{$N$} & \multirow[t]{2}{*}{$m$} & \multirow[t]{2}{*}{$w_{1}$} & \multicolumn{2}{|c|}{ \# of quest } & \multicolumn{2}{|c|}{ sol. time } & \multicolumn{2}{|c|}{ weight red. (\%) } \\
\hline & & & & avg. & $\max$ & avg. & $\max$ & avg. & $\max$ \\
\hline \multirow{12}{*}{$S W_{s}\left(a^{k}\right)=\sum_{i=1}^{m} B V\left(b_{i}^{k}\right)$} & \multirow[t]{6}{*}{25} & \multirow[t]{3}{*}{3} & 0.15 & 8.3 & 13 & 40.7 & 69.8 & 61 & 85 \\
\hline & & & 0.5 & 5.8 & 9 & 29.5 & 57.1 & 51 & 90 \\
\hline & & & 0.85 & 5.4 & 9 & 24.6 & 54.9 & 52 & 80 \\
\hline & & \multirow[t]{3}{*}{5} & 0.15 & 9.9 & 13 & 44.2 & 95.0 & 73 & 95 \\
\hline & & & 0.5 & 11.1 & 15 & 83.8 & 127.2 & 56 & 90 \\
\hline & & & 0.85 & 10.2 & 13 & 61.9 & 126.7 & 68 & 90 \\
\hline & \multirow[t]{6}{*}{50} & \multirow[t]{3}{*}{3} & 0.15 & 6 & 9 & 43.38 & 93.82 & 64 & 85 \\
\hline & & & 0.5 & 5.7 & 9 & 43.7 & 91.89 & 58 & 90 \\
\hline & & & 0.85 & 6.8 & 10 & 49.05 & 94.4 & 60 & 95 \\
\hline & & \multirow[t]{3}{*}{5} & 0.15 & 15.4 & 24 & 304.3 & 634.1 & 74 & 95 \\
\hline & & & 0.5 & 15.3 & 20 & 376.6 & 697.0 & 80 & 100 \\
\hline & & & 0.85 & 14.9 & 18 & 307.0 & 676 & 65 & 95 \\
\hline \multirow[t]{12}{*}{$S W_{m}\left(a^{k}\right)=\min _{\forall i \in I} B V\left(b_{i}^{k}\right)$} & \multirow[t]{6}{*}{25} & \multirow[t]{3}{*}{3} & 0.15 & 8 & 11 & 38.4 & 68.7 & 64 & 85 \\
\hline & & & 0.5 & 6.3 & 9 & 31.2 & 52 & 58 & 95 \\
\hline & & & 0.85 & 6 & 10 & 29.5 & 56.3 & 46 & 65 \\
\hline & & \multirow[t]{3}{*}{5} & 0.15 & 11 & 15 & 64.9 & 122.4 & 70 & 90 \\
\hline & & & 0.5 & 10.9 & 14 & 83.9 & 144.1 & 69 & 95 \\
\hline & & & 0.85 & 11 & 14 & 68.0 & 111.3 & 61 & 100 \\
\hline & \multirow[t]{6}{*}{50} & \multirow[t]{3}{*}{3} & 0.15 & 6.2 & 9 & 41.09 & 73.19 & 63 & 95 \\
\hline & & & 0.5 & 6.9 & 11 & 59.5 & 102.4 & 66 & 95 \\
\hline & & & 0.85 & 7.8 & 11 & 65.1 & 100.9 & 61 & 95 \\
\hline & & \multirow[t]{3}{*}{5} & 0.15 & 16.3 & 20 & 275.9 & 637.7 & 77 & 100 \\
\hline & & & 0.5 & 15.4 & 19 & 339.7 & 634.6 & 77 & 95 \\
\hline & & & 0.85 & 14.9 & 17 & 237.7 & 483.6 & 75 & 90 \\
\hline \multirow{12}{*}{$S W_{p}\left(a^{k}\right)=\prod_{i=1}^{m} B V\left(b_{i}^{k}\right)$} & \multirow[t]{6}{*}{25} & \multirow[t]{3}{*}{3} & 0.15 & 8.3 & 12 & 38.8 & 67.48 & 58 & 75 \\
\hline & & & 0.5 & 5.9 & 9 & 32.6 & 59.8 & 50 & 90 \\
\hline & & & 0.85 & 5.9 & 10 & 29.3 & 68.1 & 49 & 70 \\
\hline & & \multirow[t]{3}{*}{5} & 0.15 & 11.2 & 15 & 77.5 & 123.3 & 62 & 80 \\
\hline & & & 0.5 & 11.2 & 14 & 93.5 & 139.1 & 62 & 90 \\
\hline & & & 0.85 & 10.3 & 13 & 70.9 & 127.5 & 65 & 85 \\
\hline & 50 & 3 & 0.15 & 6.8 & 10 & 57.3 & 110.8 & 59 & 80 \\
\hline & & & 0.5 & 6.3 & 9 & 58.5 & 112.6 & 56 & 85 \\
\hline & & & 0.85 & 6.1 & 9 & 43.5 & 79.8 & 64 & 85 \\
\hline & & 5 & 0.15 & 17.2 & 24 & 377.1 & 666.7 & 63 & 75 \\
\hline & & & 0.5 & 16.4 & 20 & 394.0 & 681.6 & 71 & 90 \\
\hline & & & 0.85 & 15.6 & 18 & 337.6 & 667.4 & 64 & 80 \\
\hline
\end{tabular}

Table 5

Convex cone results for sum of pairwise minima and ordered weighted averaging (OWA) social welfare functions.

\begin{tabular}{|c|c|c|c|c|c|c|c|c|c|}
\hline \multirow[t]{2}{*}{ SWF form } & \multirow[t]{2}{*}{$N$} & \multirow[t]{2}{*}{$m$} & \multirow[t]{2}{*}{$w_{1}$} & \multicolumn{2}{|c|}{$\#$ of quest } & \multicolumn{2}{|c|}{ sol. time } & \multicolumn{2}{|c|}{ weight red.(\%) } \\
\hline & & & & avg. & $\max$ & avg. & $\max$ & avg. & $\max$ \\
\hline \multirow{12}{*}{$S W_{s p}\left(a^{k}\right)=\sum_{\substack{i, i^{\prime} \in I \\
\text { s.t. } \\
i \neq i^{\prime}}} \min \left(B V\left(b_{i}^{k}\right), B V\left(b_{i^{\prime}}^{k}\right)\right)$} & 25 & 3 & 0.15 & 8.2 & 11 & 42.0 & 69.7 & 58 & 75 \\
\hline & & & 0.5 & 6.5 & 9 & 38.7 & 60.6 & 51 & 95 \\
\hline & & & 0.85 & 6 & 10 & 39.2 & 84.7 & 46 & 65 \\
\hline & & 5 & 0.15 & 10.9 & 13 & 92.0 & 146.8 & 66 & 80 \\
\hline & & & 0.5 & 11.3 & 14 & 87.8 & 124.5 & 53 & 95 \\
\hline & & & 0.85 & 10.6 & 13 & 62.8 & 111.4 & 65 & 90 \\
\hline & 50 & 3 & 0.15 & 6.3 & 10 & 50.4 & 78.4 & 61 & 80 \\
\hline & & & 0.5 & 7.4 & 11 & 75.2 & 112.7 & 55 & 80 \\
\hline & & & 0.85 & 7.8 & 11 & 67.0 & 105.9 & 55 & 80 \\
\hline & & 5 & 0.15 & 17.6 & 24 & 532.3 & 843.6 & 61 & 75 \\
\hline & & & 0.5 & 16.3 & 20 & 805.6 & 1329.8 & 64 & 85 \\
\hline & & & 0.85 & 15.7 & 18 & 541.9 & 1176.1 & 63 & 80 \\
\hline \multirow{12}{*}{$S W_{o}\left(a^{k}\right)=\sum_{i=1}^{m} w_{i}^{o w a} \vec{B}_{i}^{k}$} & 25 & 3 & 0.15 & 8.3 & 12 & 39.0 & 69.0 & 58 & 75 \\
\hline & & & 0.5 & 6.7 & 9 & 33.6 & 56.9 & 47 & 80 \\
\hline & & & 0.85 & 5.9 & 10 & 34.6 & 73.8 & 46 & 65 \\
\hline & & 5 & 0.15 & 10.9 & 15 & 78.3 & 113.9 & 66 & 80 \\
\hline & & & 0.5 & 11.3 & 14 & 93.9 & 129.9 & 53 & 95 \\
\hline & & & 0.85 & 10.6 & 13 & 59.9 & 103.6 & 66 & 90 \\
\hline & 50 & 3 & 0.15 & 6.2 & 9 & 43.8 & 80.2 & 61 & 80 \\
\hline & & & 0.5 & 7.3 & 11 & 70.6 & 105.8 & 57 & 80 \\
\hline & & & 0.85 & 6.8 & 10 & 60.8 & 111.6 & 59 & 80 \\
\hline & & 5 & 0.15 & 17.6 & 24 & 385.4 & 638.7 & 61 & 75 \\
\hline & & & 0.5 & 16.4 & 20 & 424.9 & 731.2 & 65 & 90 \\
\hline & & & 0.85 & 15.7 & 18 & 329.5 & 632.4 & 63 & 80 \\
\hline
\end{tabular}


Table 6

Results of the equity index based algorithm.

\begin{tabular}{|c|c|c|c|c|c|c|}
\hline \multirow[t]{2}{*}{$N$} & \multirow[t]{2}{*}{$m$} & \multirow[t]{2}{*}{$\alpha$} & \multicolumn{2}{|c|}{ Num. of questions } & \multicolumn{2}{|c|}{ Solution Time } \\
\hline & & & avg. & $\max$ & avg & $\max$ \\
\hline \multirow[t]{6}{*}{25} & 3 & 0 & 70.4 & 85 & 135.71 & 252.66 \\
\hline & & 0.15 & 39 & 68 & 52.77 & 81.40 \\
\hline & & 0.3 & 15.8 & 38 & 6.52 & 13.29 \\
\hline & 5 & 0 & 58 & 82 & 63.49 & 127.27 \\
\hline & & 0.15 & 32 & 58 & 15.75 & 38.17 \\
\hline & & 0.3 & 17.4 & 23 & 36.71 & 59.67 \\
\hline \multirow[t]{6}{*}{50} & 3 & 0 & 34.2 & 61 & 90.49 & 188.27 \\
\hline & & 0.15 & 23.6 & 32 & 38.20 & 68.87 \\
\hline & & 0.3 & 2.4 & 5 & 0.82 & 1.21 \\
\hline & 5 & 0 & 33 & 41 & 14.47 & 20.39 \\
\hline & & 0.15 & 14.2 & 31 & 11.02 & 18.66 \\
\hline & & 0.3 & 16.2 & 35 & 20.69 & 45.89 \\
\hline
\end{tabular}

Table 7

Detailed analysis of the results for the $m=3 N=25$ instances.

\begin{tabular}{lllllll}
\hline Instance & $\alpha$ & $\begin{array}{l}\text { Alternative } \\
\text { index }\end{array}$ & $\begin{array}{l}\text { Total } \\
\text { output }\end{array}$ & $\begin{array}{l}\text { Min } \\
\text { output }\end{array}$ & $\begin{array}{l}\text { SW } \\
\text { score }\end{array}$ & $\begin{array}{l}\text { Min } \\
M V_{i j}\end{array}$ \\
\hline 1 & 0 & 11 & 436 & 10 & 4.26 & 0.00 \\
& 0.15 & 22 & 424 & 14 & 4.13 & 0.04 \\
& 0.3 & 14 & 332 & 19 & 3.08 & 0.10 \\
2 & 0 & 4 & 490 & 32 & 4.80 & 0.24 \\
& $0.15,0.3$ & 6 & 460 & 39 & 4.46 & 0.31 \\
3 & $0,0.15,0.3$ & 1 & 464 & 48 & 4.48 & 0.40 \\
4 & $0,0.15$ & 19 & 477 & 54 & 4.66 & 0.49 \\
& 0.3 & 23 & 459 & 53 & 4.46 & 0.48 \\
5 & 0 & 6 & 453 & 26 & 4.28 & 0.13 \\
& 0.15 & 13 & 445 & 44 & 4.17 & 0.33 \\
& 0.3 & 2 & 431 & 57 & 4.01 & 0.48 \\
\hline
\end{tabular}

Table 8

Underlying MVFs used in the experiments for the user specific UTA-based algorithm.

\begin{tabular}{|c|c|c|c|c|}
\hline & \multicolumn{2}{|l|}{ Set 1} & \multicolumn{2}{|l|}{ Set 2} \\
\hline & Output 1 & Output 2 & Output 1 & Output 2 \\
\hline User 1 & $\sqrt{1.5 a_{11}^{k}}$ & $\sqrt{1.5 a_{12}^{k}}$ & $\sqrt{a_{11}^{k}}$ & $\sqrt{a_{21}^{k}}$ \\
\hline User 2 & $\sqrt{2 a_{21}^{k}}$ & $\sqrt{a_{22}^{k}}$ & $1-e^{-0.05 a_{21}^{k}}$ & $1-e^{-0.06 a_{22}^{k}}$ \\
\hline User 3 & $\sqrt{a_{31}^{k}}$ & $\sqrt{2 a_{32}^{k}}$ & $1-e^{-0.01 a_{31}^{k}}$ & $1-e^{-0.02 a_{32}^{k}}$ \\
\hline User 4 & $\sqrt{2.5 a_{41}^{k}}$ & $\sqrt{0.5 a_{42}^{k}}$ & $1-e^{-0.03 a_{41}^{k}}$ & $1-e^{-0.04 a_{42}^{k}}$ \\
\hline User 5 & $\sqrt{0.5 a_{51}^{k}}$ & $\sqrt{2.5 a_{52}^{k}}$ & $a_{51}^{k}$ & $a_{52}^{k}$ \\
\hline
\end{tabular}

value function from initial interval [0-1] based on the preference information. In these experiments we used the question selection strategy that chooses two closest alternatives to an ideal alternative, due to its superior performance in the experiments on the UTA-based algorithm.

It is seen in Tables 3-5 that the number of questions asked in the convex cone based algorithm tends to be higher compared to the UTA-based algorithm. However, the convex cones based approach is more accurate (robust) in the sense that the true best alternative is always returned. The accuracy and the general per- formance of the UTA-based algorithm depends on how the parameters are chosen. A parameter set reducing the size of the set of possible marginal value functions would make more eliminations at each iteration; hence the algorithm would terminate with relatively less number of questions, sometimes at the expense of accuracy. The convex cones based approach considers a larger set of social welfare function forms hence more questions are asked to reach a conclusion.

We also observe that both approaches are satisfactory in terms of solution time as linear programming models of small-sizes are solved. It is also observed that when the number of users increases to 5 , the number of questions asked in both algorithms increases with a few exceptions.

We now discuss the results of the computational experiments performed to see the performance of the extensions of the UTAbased algorithm, which are discussed in Section 4. Table 6 summarizes the results of our experiments on the equity index based algorithm, in which we try to determine the social welfare maximizing solution that satisfies equity constraints based on a predetermined threshold $\alpha$. For each parameter setting ( $N$ and $m$ combination), we solved 5 problem instances three times, each time with a different $\alpha$ value. We use the instances that we generate for testing the performance of the UTA-based and convex cone based approaches and select the comparison questions based on their distance to an ideal point as before. It is seen that the number of questions asked and solution time decrease as $\alpha$ increases.

In Table 7, we report detailed results of the problem instances with $m=3$ and $N=25$. For each instance and equity threshold level $\alpha$, we report the alternative returned as the best solution, its total and minimum output levels, the corresponding social welfare score (calculated based on the underlying marginal value functions) and the minimum marginal value over all users and outputs. The trade-off between efficiency (SW score) and equity (the minimum marginal value score in the chosen alternative) can be seen in the results. The algorithm returns different solutions as $\alpha$ changes, with a few exceptions. It can be seen that as $\alpha$ increases, the recommended alternatives become more equitable but this comes at the expense of efficiency. We observe a similar behavior in the other problem instances that are used in the computational experiments.

We also perform computational experiments to see how the user-specific UTA based algorithm would work. Recall that in this approach, the assumption that the bundle value functions are the same over all users is relaxed. Therefore, the algorithm asks bundle comparisons to all the users and incorporates this preference information into the models. In this approach we used two sets of marginal value functions to simulate the answers to the comparison questions as summarized in Table 8 . We then normalized the obtained values to make sure that the marginal value range for each output-user pair is between 0 and 1. Table 9 summarizes the results of our experiments. We observe that this extension returns different alternatives than the UTA-based approach in most of the test instances, as expected. We also observe an increase in the number of questions asked and the solution time.

Table 9

Results of the user specific UTA-based algorithm.

\begin{tabular}{|c|c|c|c|c|c|c|c|c|c|c|c|}
\hline \multirow[t]{3}{*}{$N$} & \multirow[t]{3}{*}{$m$} & \multicolumn{5}{|c|}{ Set 1} & \multicolumn{5}{|c|}{ Set 2} \\
\hline & & \multicolumn{2}{|c|}{ \# of questions } & \multicolumn{2}{|c|}{ solution time } & \multirow[b]{2}{*}{ accuracy } & \multicolumn{2}{|c|}{ \# of questions } & \multicolumn{2}{|c|}{ solution time } & \multirow[b]{2}{*}{ accuracy } \\
\hline & & avg. & $\max$ & avg. & $\max$ & & avg. & $\max$ & avg. & $\max$ & \\
\hline \multirow[t]{2}{*}{25} & 3 & 11.8 & 32 & 50.86 & 73.12 & $80 \%$ & 12.6 & 29 & 68.57 & 79.64 & $80 \%$ \\
\hline & 5 & 11.4 & 28 & 74.61 & 139.50 & $80 \%$ & 13.4 & 24 & 137.71 & 227.44 & $80 \%$ \\
\hline \multirow[t]{2}{*}{50} & 3 & 7.4 & 23 & 54.78 & 167.85 & $80 \%$ & 6.2 & 13 & 67.41 & 158.29 & $80 \%$ \\
\hline & 5 & 5.2 & 15 & 212.16 & 305.93 & $80 \%$ & 6.4 & 12 & 310.30 & 571.84 & $100 \%$ \\
\hline
\end{tabular}




\section{Conclusion}

In this study we consider multicriteria evaluation problems in which a decision maker has to choose the best alternative (or a small subset of most preferred alternatives) among a given set of alternatives. Each alternative represents the allocation of multiple types of outputs to multiple users and is associated with a matrix, whose columns and rows correspond to outputs and users, respectively. This problem is an extension of the classical multiple criteria choice problem, in which alternatives are vectors. Moreover, since there are multiple users, equity in the distribution of the outputs across the users is important as well as efficiency. In that sense, the problem is an extension of the allocation problems that focus on the distribution of a single output.

We design two interactive algorithms that will guide the decision maker to her most preferred alternative. The first algorithm assumes additivity in the social welfare function, while in the second one we (partially) relax this assumption by defining the social welfare as a symmetric quasi-concave function of the bundle values. The fairness concerns imply special axioms for the underlying preference model of the decision maker such as impartiality, which means that the identities of the users are not important and do not affect the decision, making the problem and the solution approaches different than their counterparts in the classical multicriteria decision making literature. In the UTA-based algorithm we check whether an alternative can be better than another for all alternative pairs given the preference information via linear programming models. We then reduce the set of alternatives that are candidates to be the most preferred alternative. The second approach is based on an extension of the well-known convex cones approach. Due to the fairness concerns, the method uses generalized Lorenz dominance instead of vector dominance while checking cone dominance. This method checks dominance over the whole set of possible parameters (weights) to make robust conclusions.

We demonstrate the computational feasibility of our approaches by conducting experiments on randomly generated problem instances. We observe that the UTA-based algorithm asks less questions but this may come at the expense of accuracy. Both algorithms show satisfactory performance in terms of solution time, however they are not directly comparable since they use different comparison questions.

We also propose two extensions of the UTA-based approach: the first one offers an alternative way to model the tradeoff between efficiency and equity. This approach aims to find the most efficient solution that satisfies a predetermined equity constraint. The second extension considers the case where bundle (and marginal) value functions represent subjective judgments of the users rather than a central decision maker's, hence relaxes the assumption that they are the same over all users. We perform computational experiments to demonstrate the computational performances of these approaches and compare them to the UTAbased algorithm whenever possible. Future research may consider another hybrid approach, where equity constraints are used to make the trade-off between efficiency (sum of user utilities) and fairness more explicit as in the first extension, and where marginal value functions are defined for each output-user pair to form evaluation of alternatives from each beneficiary's point of view, as in the second extension.

As the problem is relevant in many real life decision making settings, more research in this topic awaits further attention. Future research could be performed in a few directions: In the convex cone based approach, we considered problems where the number of outputs and the number of users are not too large. Increasing the number of outputs would significantly affect the solution times due to the discretization process. Further research could be performed for developing algorithms for larger problem instances. One can also consider the multicriteria design version of this problem, in which the alternatives are implicitly defined by constraints rather than given explicitly.

\section{Acknowledgment}

We thank the Associate Editor and the anonymous referees for their thorough feedback and constructive comments, which led to substantial improvement of the manuscript. This study was supported by TUBITAK (The Scientific and Technological Research Council of Turkey) under Grant no.:215M713.

\section{References}

[1] Stewart TJ. A critical survey on the status of multiple criteria decision making theory and practice. Omega 1992;20(5-6):569-86.

[2] Diakoulaki D, Antunes CH, Martins AG. MCDA and energy planning. In: Figueira JR, Greco S, Ehrgott M, editors. Multiple criteria decision analysis: state of the art surveys. New York: Springer; 2005. p. 859-97.

[3] Marinakis V, Doukas H, Xidonas P, Zopounidis C. Multicriteria decision support in local energy planning: an evaluation of alternative scenarios for the sustainable energy action plan. Omega 2017;69:1-16. doi:10.1016/j.omega.2016.07. 005. http://www.sciencedirect.com/science/article/pii/S0305048316304492.

[4] Spronk J, Steuer RE, Zopounidis C. Multicriteria decision aid/analysis in finance. In: Greco S, Ehrgott M, Figueira JR, editors. Multiple criteria decision analysis: state of the art surveys. New York: Springer; 2016. p. 1011-65.

[5] Chiou H-K, Tzeng G-H, Cheng D-C. Evaluating sustainable fishing development strategies using fuzzy MCDM approach. Omega 2005;33(3):223-34.

[6] Mulliner E, Malys N, Maliene V. Comparative analysis of MCDM methods for the assessment of sustainable housing affordability. Omega 2016;59:146-56. doi:10.1016/j.omega.2015.05.013. http://www.sciencedirect.com/science/article/ pii/S0305048315001243.

[7] Karsu Ö. Using holistic multicriteria assessments: the convex cones approach. Wiley Encycl Oper Res Manage Sci 2013.

[8] Karsu Ö, Morton A. Inequity averse optimization in operational research. Eur Oper Res 2015;245(2):343-59.

[9] Karsu Ö. Approaches for inequity-averse sorting. Comput Oper Res 2016;66:67-80.

[10] Kostreva MM, Ogryczak W, Wierzbicki A. Equitable aggregations and multiple criteria analysis. Eur J Oper Res 2004;158(2):362-77.

[11] Ogryczak W, Wierzbicki A, Milewski M. A multi-criteria approach to fair and efficient bandwidth allocation. Omega 2008;36(3):451-63.

[12] Megiddo I, Colson AR, Nandi A, Chatterjee S, Prinja S, Khera A, et al. Analysis of the universal immunization programme and introduction of a rotavirus vaccine in India with IndiaSim. Vaccine 2014;32:A151-61.

[13] Cromwell I, Peacock SJ, Mitton C. 'Real-world' health care priority setting using explicit decision criteria: a systematic review of the literature. BMC Health Serv Res 2015;15(1):164.

[14] Dyer JS, Sarin RK. Group preference aggregation rules based on strength of preference. Manage Sci 1979;25(9):822-32.

[15] Vetschera R, Sarabando P, Dias L. Levels of incomplete information in group decision models-a comprehensive simulation study. Comput Oper Res 2014:51:160-71.

[16] Fleming M. A cardinal concept of welfare. Q J Econ 1952;66(3):366-84.

[17] Harsanyi JC. Cardinal welfare, individualistic ethics, and interpersonal comparisons of utility. J Political Economy 1955;63(4):309-21.

[18] Keeney RL, Kirkwood CW. Group decision making using cardinal social welfare functions. Manage Sci 1975;22(4):430-7.

[19] Keeney RL. A group preference axiomatization with cardinal utility. Manage Sci $1976 ; 23(2): 140-5$

[20] Greco S, Kadziński M, Mousseau V, Słowiński R. Robust ordinal regression for multiple criteria group decision: UTA-GMS-GROUP and UTADIS-GMS-GROUP. Decis Support Syst 2012;52(3):549-61.

[21] Smith JE, Von Winterfeldt D. Anniversary article: decision analysis in management science. Manage Sci 2004;50(5):561-74

[22] Eliashberg J, Winkler RL. Risk sharing and group decision making. Manage Sci $1981 ; 27(11): 1221-35$

[23] Keeney RL. Utility functions for equity and public risk. Manage Sci 1980;26(4):345-53

[24] Harvey CM. Decision analysis models for social attitudes toward inequity. Manage Sci 1985:31(10):1199-212

[25] Culyer AJ, Newhouse JP. Handbook of health economics, 1. Elsevier; 2000.

[26] Lorenz MO. Methods of measuring the concentration of wealth. Publ Am Stat Assoc 1905;9(70):209-19.

[27] Shorrocks AF. Ranking income distributions. Economica 1983;50(197):3-17.

[28] ncollection Trannoy A..Multidimensional egalitarianism and the dominance approach: a lost paradise.Inequality and economic integrationRoutledge, London2006;::284-302.

[29] Kolm S-C. Multidimensional egalitarianisms. Q J Econ 1977:1-13.

[30] Greco S, Figueira JR, Ehrgott M. Multiple criteria decision analysis: state of the art surveys. International series in operations research \& management science, 233. Springer-Verlag New York; 2016. 
[31] Jacquet-Lagrèze E, Siskos J. Une Méthode de cons truction d'une fonction d'utilité additive, explicative d'une préférence globale. Université Paris IXDauphine, Laboratoire d'analyse et modélisation de systèmes pour l'aide à la décision; 1978.

[32] Jacquet-Lagrèze E, Siskos J. Assessing a set of additive utility functions for multicriteria decision-making, the uta method. Eur J Oper Res 1982;10(2):151-64.

[33] Corrente S, Greco S, Kadziński M, Słowiński R. Robust ordinal regression in preference learning and ranking. Mach Learn 2013;93(2-3):381-422.

[34] Siskos Y, Grigoroudis E, Matsatsinis NF. UTA methods. In: Multiple criteria decision analysis: state of the art surveys. Springer; 2005. p. 297-334.

[35] Doumpos M, Zopounidis C. Multicriteria decision aid classification methods, 73. Springer Science \& Business Media; 2002.

[36] Greco S, Mousseau V, Słowiński R. Ordinal regression revisited: multiple criteria ranking using a set of additive value functions. Eur J Oper Res 2008;191(2):416-36.

[37] Greco S, Słowiński R, Figueira JR, Mousseau V. Robust ordinal regression. In: Ehrgott M, Figueira JR, Greco S, editors. Trends in multiple criteria decision analysis. New York, USA: Springer Science + Business Media, Inc; 2010. p. $241-84$.

[38] Kirkwood CW, Sarin RK. Ranking with partial information: a method and an application. Oper Res 1985;33(1):38-48.

[39] Köksalan MM, Taner OV. An approach for finding the most preferred alternative in the presence of multiple criteria. Eur J Oper Res 1992;60(1):52-60.

[40] Belton V, Stewart T. Multiple criteria decision analysis: an integrated approach. Springer Science \& Business Media; 2002.

[41] Devaud J, Groussaud G, Jacquet-Lagreze E. UTADIS: Une méthode de construction de fonctions d'utilité additives rendant compte de jugements globaux. European Working Group on Multicriteria Decision Aid, Bochum; 1980.

[42] e Costa CAB, Vansnick J-C. MACBETH-an interactive path towards the construction of cardinal value functions. Int Trans Oper Res 1994;1(4):489-500.

[43] Branke J, Corrente S, Greco S, Gutjahr W. Efficient pairwise preference elicitation allowing for indifference. Comput Oper Res 2017;88:175-86.

[44] Rothschild M, Stiglitz JE. Some further results on the measurement of inequality. J Econ Theory 1973;6(2):188-204.
[45] Dasgupta P, Sen A, Starrett D. Notes on the measurement of inequality. J Econ Theory 1973;6(2):180-7.

[46] Sen (1973) A. On economic inequality. Expanded edition with a substantial annexe by J.E. Foster and A.K. Sen. Oxford: Clarendon Press; 1997.

[47] Greco S, Mousseau V, Słowiński R. Robust ordinal regression for value functions handling interacting criteria. Eur J Oper Res 2014;239(3):711-30. doi:10.1016/j.ejor.2014.05.022. http://www.sciencedirect.com/science/article/ pii/S0377221714004457.

[48] Hazen GB. Preference convex unanimity in multiple criteria decision making. Math Oper Res 1983;8(4):505-16.

[49] Korhonen P, Wallenius J, Zionts S. Solving the discrete multiple criteria problem using convex cones. Manage Sci 1984;30(11):1336-45.

[50] Köksalan MM, Karwan MH, Zionts S. An improved method for solving multiple criteria problems involving discrete alternatives. IEEE Trans Syst Man Cybern 1984(1):24-34.

[51] Malakooti B. Theories and an exact interactive paired-comparison approach for discrete multiple-criteria problems. IEEE Trans Syst Man Cybern 1989;19(2):365-78.

[52] Taner OV, Köksalan MM. Experiments and an improved method for solving the discrete alternative multiple-criteria problem. J Oper Res Soc 1991;42(5):383-91.

[53] Özlem K, Morton A, Argyris N. Capturing preferences for inequality aversion in decision support. Eur J Oper Res 2018;264(2):686-706. doi:10.1016/j.ejor.2017. 07.018. http://www.sciencedirect.com/science/article/pii/S0377221717306458.

[54] Ogryczak W, Śliwiński T. On solving linear programs with the ordered weighted averaging objective. Eur J Oper Res 2003;148(1):80-91.

[55] Rawls J. A theory of justice. Harvard, MA: Harvard Unlversity Press, i 1971;971

[56] González-Pachón J, Romero C. Bentham, Marx and Rawls ethical principles: in search for a compromise. Omega 2016;62:47-51. doi:10.1016/j.omega.2015.08. 008. http://www.sciencedirect.com/science/article/pii/S0305048315001632.

[57] Yager RR. On ordered weighted averaging aggregation operators in multicriteria decisionmaking. IEEE Trans Syst Man Cybern 1988;18(1):183-90. 